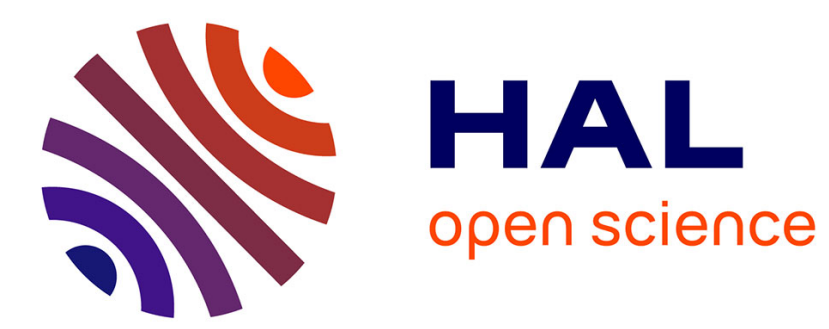

\title{
Linear stability of Taylor-Couette flow of shear-thinning fluids: modal and non-modal approaches
}

\author{
Y. Agbessi, B. Alibenyahia, Chérif Nouar, C. Lemaitre, L. Choplin
}

\section{To cite this version:}

Y. Agbessi, B. Alibenyahia, Chérif Nouar, C. Lemaitre, L. Choplin. Linear stability of Taylor-Couette flow of shear-thinning fluids: modal and non-modal approaches. Journal of Fluid Mechanics, 2015, 10.1017/jfm.2015.326 . hal-02916859

\section{HAL Id: hal-02916859 \\ https://hal.science/hal-02916859}

Submitted on 18 Aug 2020

HAL is a multi-disciplinary open access archive for the deposit and dissemination of scientific research documents, whether they are published or not. The documents may come from teaching and research institutions in France or abroad, or from public or private research centers.
L'archive ouverte pluridisciplinaire HAL, est destinée au dépôt et à la diffusion de documents scientifiques de niveau recherche, publiés ou non, émanant des établissements d'enseignement et de recherche français ou étrangers, des laboratoires publics ou privés. 


\section{Linear stability of Taylor-Couette flow of shear-thinning fluids: modal and non-modal \\ approaches}

Y. AGBESSI ${ }^{1}$, B. ALIBENYAHIA $\mathbf{A}^{3}, \mathrm{C}$. NOU A R $\mathbf{N O}^{2} \dagger$, C. LEM A I TRE ${ }^{1}$ AND L. CHOPLIN

${ }^{1}$ LRGP, UMR 7274 (CNRS), Université de Lorraine, 1 rue Grandville, BP 20451, 54001

Nancy Cedex, France

${ }^{2}$ LEMTA, UMR 7563 (CNRS), Université de Lorraine,2, avenue de la forêt de Haye, TSA 60604, 54518 Vandoeuvre Lès Nancy Cedex, France

${ }^{3}$ LApEH, Université Saad Dahleb, Blida, Algeria

(Received ?; revised ?; accepted ?. - To be entered by editorial office)

In this paper, the receptivity of circular Couette flow of shear-thinning fluids between two infinitely long coaxial cylinders to weak disturbances is addressed. It is highlighted by pseudospectra and transient growth analysis. Power-law model and Carreau model are used to describe the rheological behavior of the fluid.

The first part of the paper deals with the asymptotic long-time behavior of threedimensional infinitesimal perturbations. Using the normal-mode approach, an eigenvalue problem is derived and solved with spectral collocation method. Extensive description and classification of eigenspectra is presented. The influence of shear-thinning effects on the critical Reynolds numbers as well as on the critical azimuthal and axial wavenumbers is analyzed.

The second part investigates the short-time behavior of the disturbance using the non-

$\dagger$ Email address for correspondence: cherif.nouar@univ-lorraine.fr 
modal approach. For the same inner and outer Reynolds numbers, the amplification of the kinetic energy perturbation is much more important with increasing shear-thinning effects. Two different mechanisms are used to explain the transient growth depending on if there is or not a stratification of the angular momentum. The scaling laws for the optimal energy amplification proposed in the literature are extended to shear-thinning fluids.

\section{Introduction}

The Taylor-Couette flow of a viscous incompressible fluid between two infinitely long coaxial cylinders rotating at different angular velocities is considered as a paradigm for the study of hydrodynamic instabilities and transition in nonlinear dynamical systems. A survey of the literature on the Taylor-Couette problem can be found in Koschmieder (1993) and Tagg (1994). The basic state, i.e. a Circular Couette Flow, CCF, is purely azimuthal and presents only a radial variation across the annular gap. The linear instability of the base flow with respect to axisymmetric perturbations is governed in the inviscid limit by the Rayleigh criterion: the CCF flow is unstable with respect to axisymmetric perturbations when the square of the angular momentum, $L^{2}$, decreases with the radius, $r$, at some radial position (Drazin \& Reid 1981). In the plane $\left(R e_{1}, R e_{2}\right)$, where $R e_{1}$ and $R e_{2}$ are respectively the inner and outer Reynolds numbers, the Rayleigh stable and unstable domains are separated by the Rayleigh line. For Newtonian fluids, the Rayleigh line is given by $R e_{2} / R e_{1}=\eta$, where $\eta=R_{1} / R_{2}$ is the radius ratio. In this case, the angular momentum is constant in the annular gap. In the Rayleigh stable domain, $d L^{2} / d r>0$ in the whole annular space. On the contrary in the unstable domain and in the counter-rotating configuration, $d L^{2} / d r$ is negative near the inner cylinder and positive near the outer one. This appears to have a great effect on the wavelength of the 
critical mode (Taylor 1923). Generalized Rayleigh criterion for non axisymmetric perturbations has been derived for a free axisymmetric vortex using a large axial wavenumber WKB approximation (Billant \& Gallaire 2005).

For a viscous fluid, the same instability mechanism applies, except that the instability occurs if the destabilizing force overpasses the viscous force on a time scale smaller than the diffusion time scale. When the outer cylinder is fixed, the angular momentum decreases from the inner to the outer cylinder. The instability of the CCF of a viscous incompressible Newtonian fluid appears via a stationary axisymmetric disturbance (Taylor 1923) in the form of toroidal counter-rotating vortices regularly spaced along the axis of the cylinders. These vortices are separated by radial inflow and outflow.

For counter-rotating configuration, $L^{2}$ decreases with the radius $r$ near the inner cylinder and increases near the outer one. It is shown from linear stability analysis that for sufficient counter-rotation of the outer cylinder, the CCF becomes unstable first to a non-axisymmetric mode (Krueger et al. 1966; Snyder 1968; Langford et al. 1988). The azimuthal wavenumber of the new flow increases as the outer Reynolds number increases (Langford et al. 1988). These spiral vortices are traveling waves in the axial direction and rotating waves in the azimuthal direction (Altmeyer et al. 2011; Tagg 1994). In the unstable Rayleigh regime and co-rotating cylinders, where $L^{2}$ decreases from the inner to the outer cylinder, the CCF becomes centrifugally unstable with respect to axisymmetric toroidal vortices.

Increasing the Reynolds number above the critical values leads to the occurrence of different patterns depending on whether both cylinders are rotating or only the inner one is rotating. Andereck et al. (1986) investigated by visualization the diversity of flow states depending on the inner and outer Reynolds numbers. They identified approximately 20 flow types displayed in the plane $\left(R e_{1}, R e_{2}\right)$. When the outer cylinder is fixed, or at low 
$R e_{2}$, the transition to turbulence occurs through successive linear instabilities. The flow becomes more and more complex (Andereck et al. 1986). The transition is supercritical. For counter-rotating cylinders with sufficiently large $R e_{2}$, the subcritical nature of this transition has been shown experimentally by Coles (1965) and Atta (1966). These authors observed intermittent spiral turbulence below the linear stability boundary of the basic CCF.

The transient energy growth of a perturbation is considered as a prerequisite for a subcritical bifurcation. In this mechanism, small perturbations interact with the shear profile to create much more energetic disturbance. It is argued that despite linear stability, large growth of perturbation may trigger nonlinear effects which can sustain its energy away from zero for all times. From mathematical point of view, the transient growth is associated to the non-normality of the linear operators involved in the linear stability problem (Trefethen et al. 2000). Such effect was pointed out first by Gebhart \& Grossmann (1993) for the Taylor-Couette problem. The features of this transient growth have been investigated by Hristova et al. (2002) by the computation of the pseudospectra for axisymmetric perturbation and fixed axial periodicity, with counter-rotating cylinders such that the net effect of the Coriolis force vanishes. The authors show that the curvature increases the transient growth. Meseguer (2002) determined, for a fixed radius ratio $(\eta=0.881)$, the optimal axial and azimuthal wavenumbers which provide the maximum transient growth. The computations were performed in the linear stable regime with counter-rotating cylinders. Recently results of transient growth calculations covering all linearly stable regimes of Taylor-Couette flow were presented by Maretzke et al. (2014). To do this, they have used the shear Reynolds number and the rotation number introduced by Dubrulle et al. (2005). The authors found that optimal transient growth, in linearly stable Taylor-Couette flow, varies as $R e^{2 / 3}$ for large $R e$, suggesting that those 
effects might be significant at large $R e$.

To our knowledge, the transient growth in Taylor-Couette flow of non-Newtonian fluids has not been considered before in the literature. Actually, comparatively to Newtonian fluids, few studies have been devoted to the Taylor-Couette problem for non-Newtonian fluids despite the fact that they are involved in a wide variety of industrial applications. Their material properties have a strong impact on the flow behavior which may modify the conditions and the mechanisms of flow instabilities. Most non-Newtonian fluids have two common properties, viscoelasticity and shear-thinning. Polymer and colloid solutions as well as particulate dispersions display this behavior above a certain concentration threshold. There was a significant interest in inertialess viscoelastic Taylor-Couette instability, observed initially by Giesekus (1966) and then analyzed by Muller et al. (1989), Larson et al. (1990), Shaqfeh et al. (1992). In the laminar state, the rotation produces a shear which stretches the polymer molecules along the curved stream lines. This leads to a first normal stress-difference which acts against the centrifugal force. Groisman \& Steinberg (1998) showed experimentally that the elastic instability leads to a strong nonlinear flow transition at vanishing inertia. Hereafter, we neglect the elastic response. We focus only on the shear-thinning effects, i.e. the influence of nonlinear decrease of the effective viscosity with the shear rate. The shear-thinning behavior arises from the re-organization of the internal fluid structure in order to reduce the viscous dissipation.

\subsection{Review on Taylor-Couette flow in shear-thinning fluids}

Circular Couette flow of shear-thinning fluids are mainly characterized by viscosity stratification in the annular space. The degree of viscosity stratification defined by $\left|d \mu^{b} / d r\right|$ where $\mu^{b}$ denote the viscosity of the base flow, is even more significant as the shearthinning is more pronounced and the radius ratio $\eta$ is small. The azimuthal velocity profile is also modified: with increasing shear-thinning effects, the shear rate increases at 
the inner wall and decreases at the outer one. The mechanism of instability described for a Newtonian fluid applies for shear-thinning fluids, however, the critical conditions are modified due to the viscosity stratification and the variation of the velocity profile.

Almost all the papers dealing with the CCF of shear-thinning fluids consider the case where the inner cylinder is rotating and the outer is fixed. The critical conditions were determined experimentally or numerically for different radius ratios and different shearthinning degrees.

From an experimental point of view, Sinevic et al. (1986) determined the onset of Taylor vortices by measuring the torque exerted on the inner rotating cylinder. The results were obtained for two radius ratios $\eta=0.7$ and 0.9 . The rheological behavior of the fluids used, CMC and Carbopol solutions were described by the power-law model. Escudier et al. (1995) investigated the flow structure in a Taylor-Couette geometry with radius ratio $\eta=0.5$. The fluids used was an aqueous solution of Xanthan gum $0.15 \% \mathrm{w}$, which is shear-thinning and slightly viscoelastic and an aqueous solution of Laponite which is also shear-thinning and thixotropic. Axial and tangential velocity measurements were made using a laser Doppler anemometer. The critical Reynolds number, $R e_{c}$ defined with the inner wall shear viscosity, was determined by monitoring the development of the axial velocity component at a fixed radius with increasing the rotation speed of the inner cylinder. The authors observed that: (i) the onset of Taylor vortices is much more gradual than that for a Newtonian fluid and (ii) the shear-thinning behavior induces a significant radial shift in the location of the vortex eye towards the centerbody.

From a numerical point of view, the critical conditions were either determined using 2D direct numerical simulation (Lockett et al. 2004; Coronado-Malutti et al. 1986) or by solving a generalized eigenvalue problem which results from a linear stability analysis (Jastrzebski et al. 1992; Ashrafi \& Khayat 2000; Caton 2006). A critical review of these 
studies was done by Alibenyahia et al. (2012). It was shown in this paper that when the Reynolds number is defined using the inner-wall shear viscosity, shear-thinning delays the appearance of Taylor vortices. This delay is due to a reduction in the energy exchange between the base flow and the perturbation and not to the modification of the viscous dissipation.

In all the previous studies, the outer cylinder is fixed. The case where the two cylinders are both rotating was considered, to our knowledge, only by Pascal \& Rasmussen (1995). These authors determined the critical Reynolds number for power-law fluids at two radius ratios $\eta=0.7$ and 0.9 . The results are limited to two values of shear-thinning index (equation 2.8) $n=0.8$ and 1.2. The perturbation is assumed to be axisymmetric and the viscosity perturbation was not taken into account.

\subsection{Objectives, methodology and outline of the paper}

The objectives of the present study are to investigate the influence of the shear-thinning behavior characterized by the stratification of the viscosity and the modification of the velocity profile on a three-dimensional linear stability analysis of CCF. In this study, both cylinders are rotating independently and thin and wide gaps are considered. Short- and long-time evolution of the perturbation will be considered. Many models are available to describe the dependence of the viscosity on the shear rate. All the governing equations of the present paper are given for a general nonlinear purely viscous fluid. For numerical computations we adopt two models: (i) the power-law and (ii) the Carreau (1972) model. Power-law model is the most popular. It has the advantage to provide simple analytical expressions. However for very large annular spaces, shear rate at the vicinity of the outer cylinder could be very low and, in this situation, the power-law model is not very accurate. The Carreau model has a sound theoretical basis and is $C^{\infty}$ (differentiable for all degrees of differentiation) with respect to the second invariant of the strain-rate 
tensor $\Gamma$. The main disadvantage is that the base flow has to be calculated numerically. Note that the Carreau model approaches the power-law as the ratio of the infinite-shear viscosity $\mu_{\infty}$ to the zero-shear viscosity $\mu_{0}$ can be neglected and the characteristic time of the fluid $\lambda$ is very large.

The article is organized as follows. In section 2 , the governing equations and dimensionless parameters are given. The effect of the rheology on the base flow and on the Rayleigh line is described in section 3 and 4 . The linear perturbation equations are derived in section 5 . In section 6 , the long-time behavior of the perturbation is considered. The influence of the shear-thinning effect on the eigenspectra and critical conditions are analyzed. The linear stability problem is presented. The resulting eigenvalue problem is solved numerically. Section 7 deals with the pseudo-spectra, transient growth and optimal perturbation. Conclusions of this study are presented in section 8 .

\section{Mathematical formulation}

\subsection{Governing equations}

We consider the flow of a purely viscous non-Newtonian fluid between two infinitely long concentric cylinders. The inner cylinder of radius $\hat{R}_{1}$ is rotating with a constant angular velocity $\hat{\Omega}_{1}>0$. The outer cylinder of radius $\hat{R}_{2}$ is rotating with a constant angular velocity $\hat{\Omega}_{2}$, with $\hat{\Omega}_{2}>0$ for co-rotating cylinders and $\hat{\Omega}_{2}<0$ for counterrotating cylinders. The radius ratio is $\eta=\hat{R}_{1} / \hat{R}_{2}$. The incompressibility condition and the momentum equations are

$$
\begin{aligned}
\boldsymbol{\nabla} \cdot \boldsymbol{U} & =0 \\
\frac{\partial \boldsymbol{U}}{\partial t}+R e_{1}(\boldsymbol{U} \cdot \boldsymbol{\nabla}) \boldsymbol{U} & =-\nabla P+\nabla \cdot \boldsymbol{\tau},
\end{aligned}
$$


where $\boldsymbol{U}=U \boldsymbol{e}_{r}+V \boldsymbol{e}_{\theta}+W \boldsymbol{e}_{z}$ is the velocity vector in cylindrical coordinates $(r, \theta, z), P$ the pressure and $\tau$ the deviatoric extra-stress tensor. The quantities denoted with a hat $(\stackrel{)}{)}$ are dimensional while quantities without a hat are non-dimensional. We define the Reynolds number for the inner $R e_{1}$ and the outer $R e_{2}$ cylinders as

$$
R e_{1}=\frac{\hat{\rho} \hat{R}_{1} \hat{\Omega}_{1} \hat{d}}{\hat{\mu}_{r e f}}, \quad R e_{2}=\frac{\hat{\rho} \hat{R}_{2} \hat{\Omega}_{2} \hat{d}}{\hat{\mu}_{\text {ref }}}
$$

The governing equations have been non-dimensionalized using the width of the annular space $\hat{d}=\hat{R}_{2}-\hat{R}_{1}$ as the reference length scale, the velocity of the inner cylinder $\hat{\Omega}_{1} \hat{R}_{1}$ as the velocity scale, the viscous diffusion time $\hat{\rho} \hat{d}^{2} / \hat{\mu}_{r e f}$ for time scale and the quantity $\hat{\mu}_{r e f} \hat{R}_{1} \hat{\Omega}_{1} / \hat{d}$ for stresses and pressure scale where $\hat{\mu}_{r e f}$ is a reference viscosity which will be specified later for each rheological model. To the previous equations, we add the no-slip and impermeability conditions at the walls,

$$
\boldsymbol{U}\left(R_{1}, \theta, z\right)=(0,1,0), \quad \boldsymbol{U}\left(R_{2}, \theta, z\right)=\left(0, \frac{R e_{2}}{R e_{1}}, 0\right),
$$

where the dimensionless radii are expressed as $R_{1}=\eta /(1-\eta)$ and $R_{2}=1 /(1-\eta)$.

\subsection{Rheological models}

We consider a purely viscous shear-thinning fluid. The viscous stress tensor reads

$$
\boldsymbol{\tau}=\mu(\Gamma) \dot{\gamma} \quad \text { with } \quad \dot{\gamma}=\nabla \boldsymbol{u}+(\boldsymbol{\nabla} \boldsymbol{u})^{T}
$$

where the second invariant of the strain-rate tensor is expressed as

$$
\Gamma=\frac{1}{2} \dot{\gamma}_{i j} \dot{\gamma}_{i j}
$$

- The power-law model is given by

$$
\hat{\mu}=\hat{K} \hat{\Gamma}^{\frac{n-1}{2}}
$$


where $\hat{K}$ is the consistency, and $n \leqslant 1$ the shear-thinning index. In dimensionless form, the viscosity becomes

$$
\mu=\Gamma^{\frac{n-1}{2}}
$$

and the reference viscosity reads

$$
\hat{\mu}_{r e f}=\hat{K}\left(\hat{R}_{1} \hat{\Omega}_{1} / \hat{d}\right)^{n-1}
$$

- The Carreau model is given by

$$
\frac{\hat{\mu}-\hat{\mu}_{\infty}}{\hat{\mu}_{0}-\hat{\mu}_{\infty}}=\left(1+\hat{\lambda}^{2} \hat{\Gamma}\right)^{\frac{n-1}{2}}
$$

with $\hat{\mu}_{0}$ and $\hat{\mu}_{\infty}$ the viscosities at low and high shear rates and $\hat{\lambda}$, the characteristic time of the fluid. The location of the transition from the Newtonian plateau to the shear-thinning regime is determined by $1 / \hat{\lambda}$. The infinite-shear viscosity $\hat{\mu}_{\infty}$ is generally associated with a breakdown of the fluid, and is frequently significantly smaller $\left(10^{-3}\right.$ to $10^{-4}$ times as small) than $\hat{\mu}_{0}$, see Bird et al. (1987) and Tanner (2000). The ratio $\hat{\mu}_{\infty} / \hat{\mu}_{0}$ will thus be neglected in the following. Increasing $\hat{\lambda}$ reduces the Newtonian plateau to lower shear rates and the model thus tends to a power-law model. The dimensionless effective viscosity is then

$$
\mu=\left(1+\lambda^{2} \Gamma\right)^{\frac{n-1}{2}} \quad \text { with } \quad \lambda=\frac{\hat{\lambda} \hat{\rho} \hat{\mu}_{r e f}}{\hat{d}^{2}}
$$

and the viscosity scale reads in this case

$$
\hat{\mu}_{r e f}=\hat{\mu}_{0} .
$$

Note that the reference viscosities adopted here (2.9) and (2.12) are those widely used in the literature. 


\subsection{Governing parameters}

The dimensionless governing parameters are the rheological parameters, $n$ for the powerlaw model, $n$ and $\lambda$ for the Carreau model, the radius ratio $\eta$ and the inner and outer Reynolds numbers $R e_{1}$ and $R e_{2}$. In order to separate shear and centrifugal effects, Dubrulle et al. (2005) introduced another parametrization replacing $R e_{1}$ and $R e_{2}$ by a shear Reynolds number $R e$, ratio between the shear time scale $\left|\hat{\dot{\gamma}}_{r \theta}^{b}(\hat{\bar{r}})\right|^{-1}$ and the viscous time scale $\hat{\rho} \hat{d}^{2} / \hat{\mu}$ and a rotation number $R_{\Omega}$, ratio between shear time scale and a rotation time scale:

$$
R e=\frac{\hat{\rho}\left|\hat{\dot{\gamma}}_{r \theta}^{b}(\hat{\bar{r}})\right| \hat{d}^{2}}{\hat{\mu}^{b}(\hat{\bar{r}})} \quad, \quad R_{\Omega}=\frac{2 \hat{\Omega}_{f}(\hat{\bar{r}})}{\hat{\dot{\gamma}}_{r \theta}^{b}(\hat{\bar{r}})}
$$

The superscript $b$ refers to the base flow which is purely azimuthal, $\hat{\dot{\gamma}}_{r \theta}^{b}(\hat{\bar{r}})$ is the base shear rate evaluated at a radial position $\hat{\bar{r}}$. Here, $\hat{\bar{r}}$ is the radial position of a frame rotating with an angular velocity $\hat{\Omega}_{f}(\hat{\bar{r}})$ such that

$$
\left[\hat{V}^{b}\left(\hat{R}_{1}\right)-\hat{\Omega}_{f} \hat{R}_{1}\right]=-\left[\hat{V}^{b}\left(\hat{R}_{2}\right)-\hat{\Omega}_{f} \hat{R}_{2}\right]
$$

With respect to this rotating frame, the cylinders are seen to rotate at exact opposite velocities. For a Newtonian fluid, it can be shown straightforwardly (Dubrulle et al. 2005) that

$$
\bar{r}=\sqrt{R_{1} R_{2}} \quad, \quad R e=\frac{2\left|\eta R e_{2}-R e_{1}\right|}{1+\eta} \quad, \quad R_{\Omega}=\frac{(1-\eta)\left(R e_{1}+R e_{2}\right)}{\eta R e_{2}-R e_{1}} .
$$

For power-law fluids, using equations (2.13) and (2.14), we obtain

$$
\bar{r}=\left(\frac{\left(R_{1}+R_{2}\right) R_{1}^{\frac{2}{n}-1} R_{2}^{\frac{2}{n}-1}}{R_{1}^{\frac{2}{n}-1}+R_{2}^{\frac{2}{n}-1}}\right)^{\frac{n}{2}} \quad, \quad R e=\frac{R e_{1}}{\left|\dot{\gamma}_{r \theta}^{b}(\bar{r})\right|^{(n-2)}} \quad, \quad R_{\Omega}=-\frac{2}{n} \frac{B_{0}}{\bar{r}^{2 / n}}
$$

where $B_{0}$ is given by equation (3.5). Figure 1 displays some particular values of $R_{\Omega}$ in the usual parameter space $\left(R e_{1}, R e_{2}\right)$ for a Newtonian fluid and for a power-law fluid with $n=0.3$. Shear-thinning effects introduce a slight deviation of constant $R_{\Omega}$ lines. For Carreau fluids, $\bar{r}, R e$, and $R_{\Omega}$ are evaluated numerically. The constant $R_{\Omega}$ lines are 

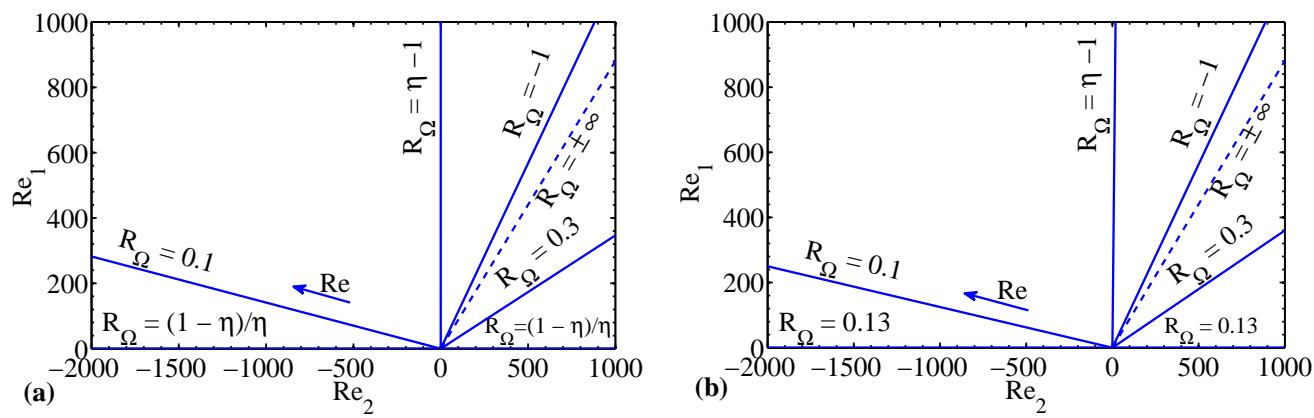

Figure 1. Parameters of Dubrulle et al. (2005) represented in the $\left(R e_{1}, R e_{2}\right)$ plane at $\eta=0.881$.

Contours of rotation number $R_{\Omega}$ for (a) Newtonian fluid and (b) power-law fluid with $n=0.3$.

The arrow indicates that $R e$ increases along the constant $R_{\Omega}$ line.

similar to those of figure $1(\mathrm{~b})$, and are therefore not represented. In the following, we have chosen to express most of our result in the $\left(R e_{1}, R e_{2}\right)$ plane, because we believe it is more understandable for the reader. In appendix D, some results for marginal stability are presented in the plane $\left(R e, R_{\Omega}\right)$. As far as the transient growth is concerned, based on the work of Maretzke et al. (2014), we will use the $\left(R e, R_{\Omega}\right)$ representation in order to obtain a scaling of the energy growth.

\section{Base flows}

The base flow is azimuthal and is solution of

$$
\frac{d}{d r}\left(r^{2} \tau_{r \theta}^{b}\right)=0
$$

with the boundary conditions

$$
V^{b}\left(R_{1}\right)=1 \quad \text { and } \quad V^{b}\left(R_{2}\right)=\frac{R e_{2}}{R e_{1}}
$$

Hereafter, we describe the velocity and the viscosity profiles for power-law and Carreau fluids in the case of a thin and a wide gap, $\eta=0.881$ and $\eta=0.4$ respectively. 


\subsection{Power-law fluid}

The velocity profile is (Bird et al. 1987)

$$
V^{b}(r)=A_{0} r+\frac{B_{0} r}{r^{2 / n}}
$$

with

$$
\begin{gathered}
A_{0}=\frac{1}{R_{1}}\left[1+\frac{1}{R_{1}^{2 / n}}\left(1-\frac{R e_{2}}{R e_{1}} \eta\right)\left(\frac{1}{R_{2}^{2 / n}}-\frac{1}{R_{1}^{2 / n}}\right)^{-1}\right] \\
B_{0}=-\frac{1}{R_{1}}\left[\left(1-\frac{R e_{2}}{R e_{1}} \eta\right)\left(\frac{1}{R_{2}^{2 / n}}-\frac{1}{R_{1}^{2 / n}}\right)^{-1}\right]
\end{gathered}
$$

Figure 2 shows the effect of the shear-thinning index on the velocity and viscosity profiles for $\eta=0.4$. With increasing shear-thinning effects, the curvature of the velocity profile becomes more pronounced, figure 2(a). Shear rate increases at the inner wall and decreases at the outer one. The wider the gap the more this effect is significant. This results in a viscosity stratification within the gap, figure 2(b). The viscosity as well as the degree of the viscosity stratification increases from the inner to the outer wall. This is more pronounced with increasing shear thinning effects (decreasing $n$ ). Figure 3 shows an example of velocity profile for co-rotating cylinders. The same characteristics as for counter-rotating cylinders are observed, except that the velocity profile is convex instead of concave.

\subsection{Carreau fluid}

Since no analytical expression of the velocity profile exists in that case, equation (3.1) is solved numerically using an iterative process combined with a Chebyshev polynomial approximation of the velocity profile. Details can be found in Alibenyahia et al. (2012). For a given value of $\lambda$, the effect of index $n$ is similar to the effect of the power-law fluid index. When increasing $\lambda$, the base flow tends to that of a power-law fluid. This is observed for values of $\lambda$ as low as $\lambda=10$ (figure 2). 

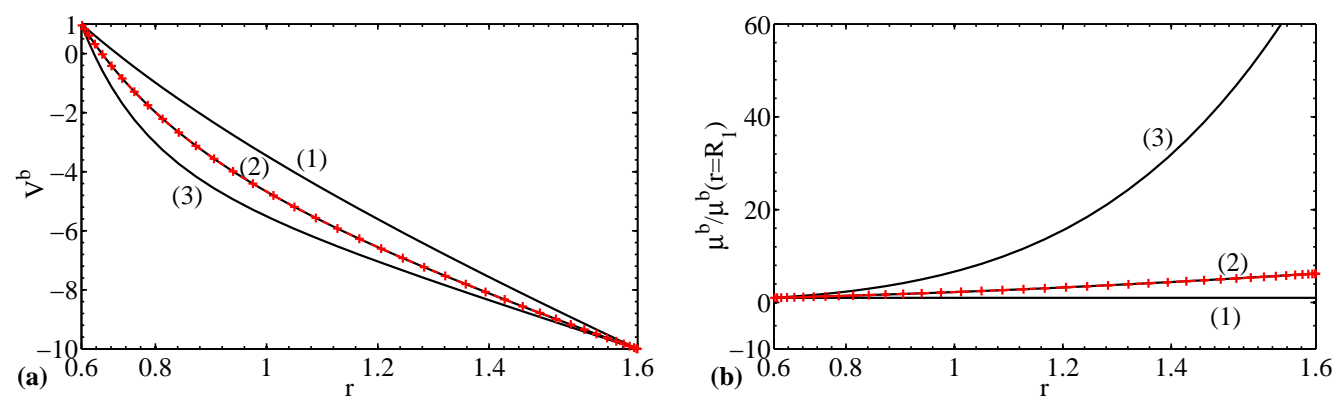

FIgURE 2. Base flow for power-law fluids in the case of counter-rotating cylinders with $\eta=0.4$, $R e_{1}=300$ and $R e_{2}=-3000$. (a) Velocity profiles and (b) viscosity profiles for (1) $n=1$ (Newtonian fluid) (2) $n=0.5$ and (3) $n=0.3 .(-+-)$ Carreau fluid with $n=0.5$ and $\lambda=10$.

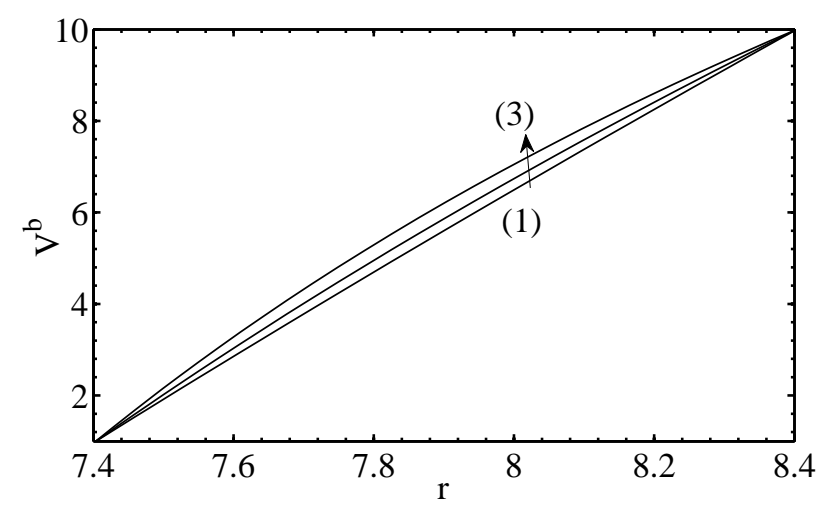

FiguRE 3. Base flow for power-law fluids in the case of co-rotating cylinders with $\eta=0.881$,

$$
R e_{1}=300 \text { and } R e_{2}=3000,(1) n=1 \text { (Newtonian fluid), (2) } n=0.5 \text { and (3) } n=0.3 .
$$

\section{Solid rotation and Rayleigh lines}

The solid rotation line corresponds to the situation where $\hat{\Omega}_{1}=\hat{\Omega}_{2}$ and $R e_{1}=\eta R e_{2}$. It is represented with dashed lines in figure 4. In the parametrization of Dubrulle et al. (2005), the solid line is given by $R_{\Omega}= \pm \infty$.

As indicated in the introduction, the instability of the Taylor-Couette flow is due to the stratification of the angular momentum. When the cylinders are co-rotating, the Rayleigh stable regime for an inviscid fluid is defined by $\frac{d}{d r}\left(L^{2}\right)>0, \forall r \in\left[R_{1}, R_{2}\right]$, where $L=r V^{b}$ is the angular momentum (Drazin \& Reid 1981). This regime is bounded 

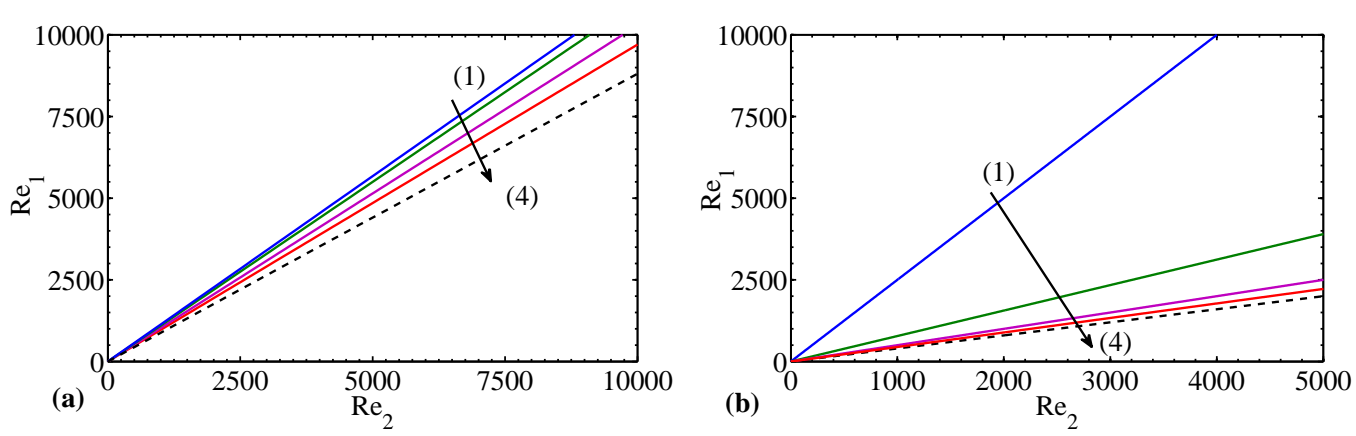

FiguRE 4. Rayleigh line for a power-law fluid with (a) $\eta=0.881$ and (b) $\eta=0.4$ : (1)

Newtonian fluid $n=1$, (2) $n=0.5$, (3) $n=0.2$ and (4) $n=0.1$. (- - -) solid rotation line.

in the $\left(R e_{1}, R e_{2}\right)$ plane by the Rayleigh line, on which $\exists r$ such that $\frac{d}{d r}\left(L^{2}\right)=0$. For a Newtonian fluid, the Rayleigh line equation reads

$$
R e_{1}=\frac{R e_{2}}{\eta}
$$

Following the same approach for the non-Newtonian models considered here, the Rayleigh criterion is derived straightforwardly.

For power-law model, we obtain

$$
R e_{1}=\left(\frac{1}{n\left(\eta^{2 / n}-1\right)+1}\right) \eta R e_{2}
$$

For the Carreau model, the Rayleigh line is computed numerically. As expected, for sufficiently large $\lambda$, say $\lambda>10$, we recover the power-law results for the same $n$. Decreasing $\lambda$ reduces shear-thinning effects and tends progressively to the Newtonian case.

As illustrated in figures 4(a)-(b), with increasing shear-thinning effects (decreasing $n$ or increasing $\lambda$ ) the Rayleigh line approaches the solid rotation line, thus reducing the socalled quasi-Keplerian zone which is delimited by these two lines in plane $\left(R e_{1}, R e_{2}\right)$. In the quasi-Keplerian regime, the angular velocity decreases radially while the angular momentum increases radially.

Note that for a Newtonian fluid, the Rayleigh line corresponds to $R_{\Omega}=-1$. For a shear- 
thinning fluid, the calculations show that the Rayleigh line deviates from $R_{\Omega}=-1$. This effect is more significant for a large gap and strong shear-thinning effects. For instance, for a power-law fluid with $n=0.3$ and $\eta=0.4$, the Rayleigh line corresponds to $R_{\Omega}=-3.17$

\section{Linear perturbation equations}

In a classical way, we superimposed to the base flow an infinitesimal perturbation

$$
\{\boldsymbol{U}, P, \boldsymbol{\tau}\}=\left\{\boldsymbol{U}^{b}, P^{b}, \boldsymbol{\tau}^{b}\right\}+\epsilon\left\{\boldsymbol{u}^{\prime}, p^{\prime}, \boldsymbol{\tau}^{\prime}\right\}
$$

Substituting $(\boldsymbol{U}, P, \boldsymbol{\tau})$ by their expression (5.1) in equations (2.1)-(2.2) and linearizing around the base flow, we obtain the perturbation equations

$$
\begin{aligned}
\boldsymbol{\nabla} \cdot \boldsymbol{u}^{\prime} & =0 \\
\frac{\partial \boldsymbol{u}^{\prime}}{\partial t}+R e_{1}\left[\left(\boldsymbol{U}^{b} \cdot \boldsymbol{\nabla}\right) \boldsymbol{u}^{\prime}+\left(\boldsymbol{u}^{\prime} \cdot \boldsymbol{\nabla}\right) \boldsymbol{U}^{\boldsymbol{b}}\right] & =-\nabla p^{\prime}+\nabla \cdot \boldsymbol{\tau}^{\prime}
\end{aligned}
$$

where

$$
\boldsymbol{\tau}^{\prime}=\frac{1}{\epsilon}\left\{\boldsymbol{\tau}\left(\boldsymbol{U}^{b}+\epsilon \boldsymbol{u}^{\prime}\right)-\boldsymbol{\tau}\left(\boldsymbol{U}^{b}\right)\right\}=\mu^{b} \dot{\gamma}\left(\boldsymbol{u}^{\prime}\right)+\left(\mu_{t}-\mu^{b}\right) \boldsymbol{A}
$$

is the perturbation of the deviatoric shear-stress tensor. The components of tensor $\boldsymbol{A}$ are all zero except for $A_{1,2}=A_{2,1}=\dot{\gamma}_{r \theta}\left(\boldsymbol{u}^{\prime}\right)$. We have introduced in equation (5.4) the tangential viscosity $\mu_{t}$

$$
\mu_{t}=\mu\left(\boldsymbol{U}^{b}\right)+\frac{\partial \mu}{\partial \dot{\gamma}_{r \theta}}\left(\boldsymbol{U}^{b}\right) \dot{\gamma}_{r \theta}\left(\boldsymbol{U}^{b}\right)
$$


We obtain the system

$$
\begin{aligned}
0 & =D_{*} u^{\prime}+\frac{1}{r} \frac{\partial v^{\prime}}{\partial \theta}+\frac{\partial w^{\prime}}{\partial z}, \\
\frac{\partial u^{\prime}}{\partial t} & =-R e_{1} \frac{V^{b}}{r}\left(\frac{\partial u^{\prime}}{\partial \theta}-2 v^{\prime}\right)-D p^{\prime}+\mu^{b}\left[\Delta u^{\prime}-2 \frac{1}{r^{2}} \frac{\partial u^{\prime}}{\partial \theta}-\frac{u^{\prime}}{r^{2}}\right] \\
& +2 D \mu^{b} D u^{\prime}+\frac{1}{r} \frac{\partial\left(\mu_{t}-\mu^{b}\right)}{\partial \theta}\left(\tilde{D} v^{\prime}+\frac{1}{r} \frac{\partial u^{\prime}}{\partial \theta}\right), \\
\frac{\partial v^{\prime}}{\partial t} & =-R e_{1}\left(u^{\prime} D_{*} V^{b}+\frac{V^{b}}{r} \frac{\partial v^{\prime}}{\partial \theta}\right)-\frac{1}{r} \frac{\partial p^{\prime}}{\partial \theta}+\mu^{b}\left[\Delta v^{\prime}+\frac{2}{r^{2}} \frac{\partial u^{\prime}}{\partial \theta}-\frac{v^{\prime}}{r^{2}}\right] \\
& +D \mu^{b}\left[D v^{\prime}-\frac{v^{\prime}}{r}+\frac{1}{r} \frac{\partial u^{\prime}}{\partial \theta}\right]+D_{*}\left[\left(\mu_{t}-\mu^{b}\right)\left(\tilde{D} v^{\prime}+\frac{1}{r} \frac{\partial u^{\prime}}{\partial \theta}\right)\right] \\
& +\left(\mu_{t}-\mu^{b}\right)\left[\frac{1}{r}\left(\tilde{D} v^{\prime}+\frac{1}{r} \frac{\partial u^{\prime}}{\partial \theta}\right)\right], \\
\frac{\partial w^{\prime}}{\partial t} & =-R e_{1} \frac{V^{b}}{r} \frac{\partial w^{\prime}}{\partial \theta}-\frac{\partial p^{\prime}}{\partial z}+\mu^{b} \Delta w^{\prime}+D \mu^{b}\left[D w^{\prime}+\frac{\partial u^{\prime}}{\partial z}\right]
\end{aligned}
$$

where $D=\frac{\partial}{\partial r}, D_{*}=D+\frac{1}{r}, \tilde{D}=D-\frac{1}{r}$ and $\Delta=D^{2}+\frac{1}{r} D+\left(\frac{1}{r} \frac{\partial}{\partial \theta}\right)^{2}+\left(\frac{\partial}{\partial z}\right)^{2}$.

The boundary conditions are obtained by assuming no slip and impermeability at inner and outer walls,

$$
\boldsymbol{u}^{\prime}=0 \quad \text { at } \quad r=R_{1}, R_{2}
$$

The linear system (5.6) - (5.9) is homogeneous in the axial and azimuthal directions. Perturbations can thus be decomposed into Fourier modes with an azimuthal wavenumber $m \in \mathbb{Z}$ and an axial wavenumber $k \in \mathbb{R}$ :

$$
\left\{\boldsymbol{u}^{\prime}, p^{\prime}\right\}=\{\tilde{\boldsymbol{u}}(r, t), \tilde{p}(r, t)\} \exp [i(m \theta+k z)]+c . c .
$$

where, c.c. stands for the complex conjugate and $\tilde{\boldsymbol{u}}(r, t)=\tilde{u}(r, t) \boldsymbol{e}_{r}+\tilde{v}(r, t) \boldsymbol{e}_{\theta}+\tilde{w}(r, t) \boldsymbol{e}_{z}$. By eliminating the pressure and one velocity component, the resulting system can be written in terms of two components of the velocity $(\tilde{u}, \tilde{v})$ for $k \neq 0$ or $(\tilde{u}, \tilde{w})$ for $m \neq 0$. The different formulations lead to an initial value problem which can be written formally as

$$
\mathcal{L} \tilde{\boldsymbol{q}}=\mathcal{M} \frac{\partial \tilde{\boldsymbol{q}}}{\partial t}
$$


where $\tilde{\boldsymbol{q}}=(\tilde{u}, \tilde{v})^{T}$ or $(\tilde{u}, \tilde{w})^{T}$ depending on the formulation adopted. The linear operators $\mathcal{L}$ and $\mathcal{M}$ are given in appendix $\mathrm{A}$.

\section{Long-time behavior of the disturbance: eigenvalue problem}

The initial value problem can be turned into an eigenvalue problem by the assumption of the exponential time dependence, i.e. $\tilde{\boldsymbol{q}}(r, t)=\boldsymbol{q}(r) \exp (\sigma t)$. The resulting generalized eigenvalue problem reads

$$
\mathcal{L} \boldsymbol{q}=\sigma \mathcal{M} \boldsymbol{q}
$$

with the complex frequency $\sigma$ as the eigenvalue. The real part of $\sigma$ is the growth rate of the perturbation and its imaginary part is its oscillations frequency. The eigenvalue problem (6.1) is discretized using a spectral collocation method based on Chebyshev polynomials evaluated at $N$ collocation points of Gauss-Lobatto. The matrix eigenvalue problem that results is solved using QZ algorithm with Matlab. To ensure the convergence of the numerical method, computations were performed for increasing $N$. It was found that for $N \geqslant 40$, the five first eigenvalues remain unchanged with three digits accuracy. Each computation was then performed for $N$ and $N+5$ and only the overlapping eigenvalues were retained. This procedure allows one to remove spurious eigenvalues.

\subsection{Eigenvalue spectra}

In this section, eigenspectra are analyzed for axisymmetric and non axisymmetric perturbations. For both situations, the inner Reynolds number is fixed and the outer Reynolds number is varied. Then, $R e_{2}$ is fixed and $R e_{1}$ is increased until the marginal stability is reached. 
6.1.1. Axisymmetric perturbation $m=0$

The perturbation is two-dimensional and the $(\tilde{u}, \tilde{v})$ formulation is used. As $m=0$, the linear operators $\mathcal{L}$ and $\mathcal{M}$, given by the equations (A 2-A 7) in the appendix $\mathrm{A}$ are real. Therefore, the eigenvalues are real or complex conjugate. Spectra for that case are presented in figure 5 for a Newtonian fluid.

On the Rayleigh line, the angular momentum is constant. The spectrum, figure 5(a), consists of a vertical branch with real eigenvalues. It is independent of $R e_{1}$, as indicated by Gebhart \& Grossmann (1993). These properties are demonstrated in appendix B.

When the outer cylinder is fixed, $R e_{2}=0$, most of the eigenvalues are real, figure $5(\mathrm{~b})$. DiPrima \& Hall (1984) have shown that the first eigenvalue that crosses the real axis is always real, in agreement with the principle of stability exchange.

For counter-rotating cylinders, the real vertical branch, which is made of paired eigenvalues, splits into two lateral curved branches, i.e. one pair of eigenvalues separates into two complex conjugate eigenvalues, figure $6(\mathrm{a})$. The higher the absolute value of $R e_{2}$, the lower the locus of the branch separation. The imaginary part of the spectra are bounded by the epicyclic frequency $\kappa$ evaluated at the outer wall, $r=R_{2}$ (Shu 1982). The epicyclic frequency is defined by

$$
\kappa^{2}(r)=\left(-\frac{1}{r^{3}} \frac{d}{d r}(r V)^{2}\right)
$$

For a Newtonian fluid at $r=R_{2}$,

$$
\kappa^{2}\left(R_{2}\right)=4 R e_{2}\left(R e_{2}-\eta R e_{1}\right)\left(\frac{1-\eta}{1+\eta}\right)
$$

Similar results are observed for a wide gap, figure $6(\mathrm{~b})$.

Additional computations not presented here were conducted for co-rotating cylinders and spectrum evolutions similar to the counter-rotating situation have been obtained.

For shear-thinning fluids, eigenspectra are quite similar to the Newtonian case. However, 

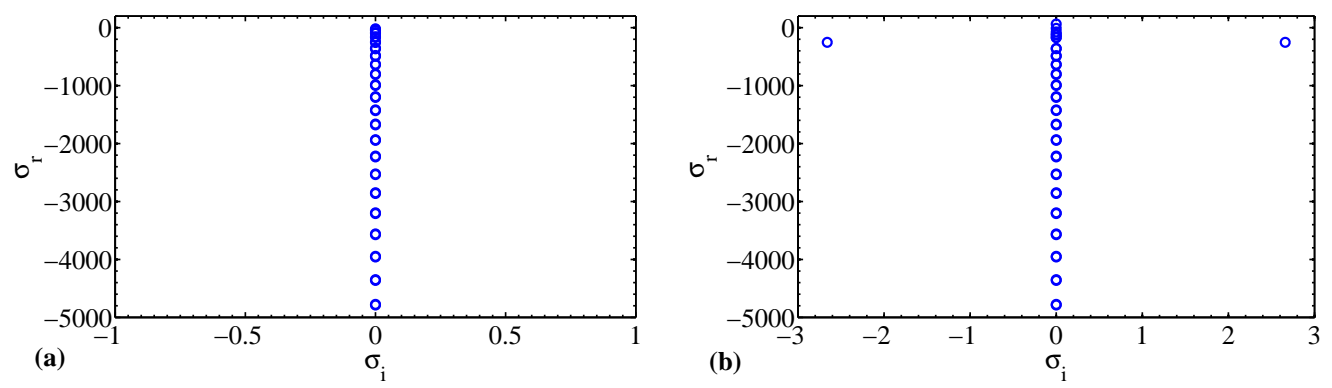

FiguRE 5. Eigenspectra for a Newtonian fluid undergoing an axisymmetric perturbation $m=0$ for $\eta=0.881, k=\pi, R e_{1}=400$, and varying $R e_{2}$. (a) $R e_{2}=352.4$ (on the Rayleigh line) and (b) $R e_{2}=0$.
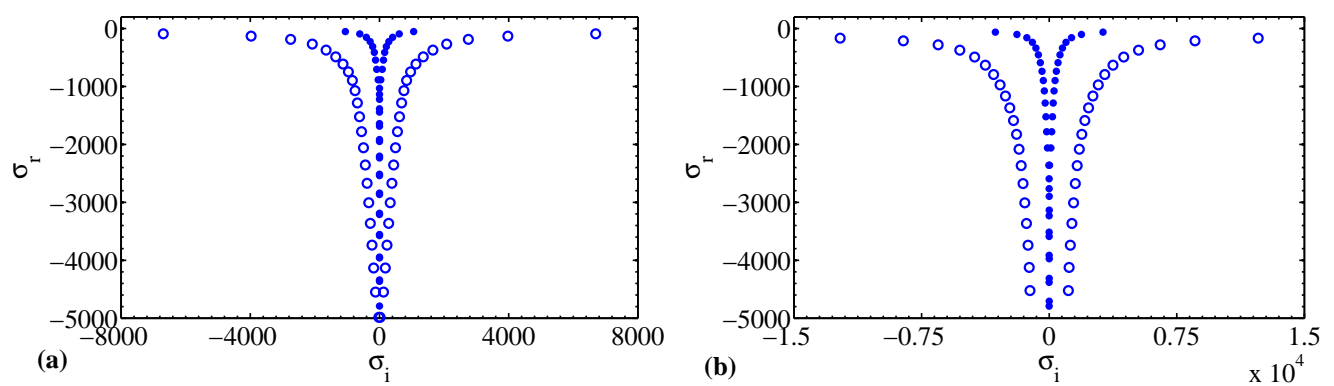

FiguRE 6. Eigenspectra for a Newtonian fluid undergoing an axisymmetric perturbation $m=0$, $k=\pi, R e_{1}=400$, and varying $R e_{2}$. (a) $\eta=0.881$, (०) $R e_{2}=-4000,(\bullet) R e_{2}=-25000$ and (b) $\eta=0.4,(\circ) R e_{2}=-4000,(\bullet) R e_{2}=-25000$.

two differences have been noted:

(i) on the Rayleigh line, the angular momentum is no longer constant in the annular space, thus the eigenvalues are not necessarily real, figure $7(\mathrm{a})$, and furthermore, they vary with $R e_{1}$.

(ii) for the same values of $R e_{1}$ and $R e_{2}$, the vertical branch separation occurs closer to the real axis than for a Newtonian fluid.

Evolution of the eigenspectra, when $R e_{2}$ is fixed and $R e_{1}$ is increased until the critical value, is displayed in figure $7(\mathrm{~b})$ for a power-law fluid with $n=0.5$. The critical mode is 

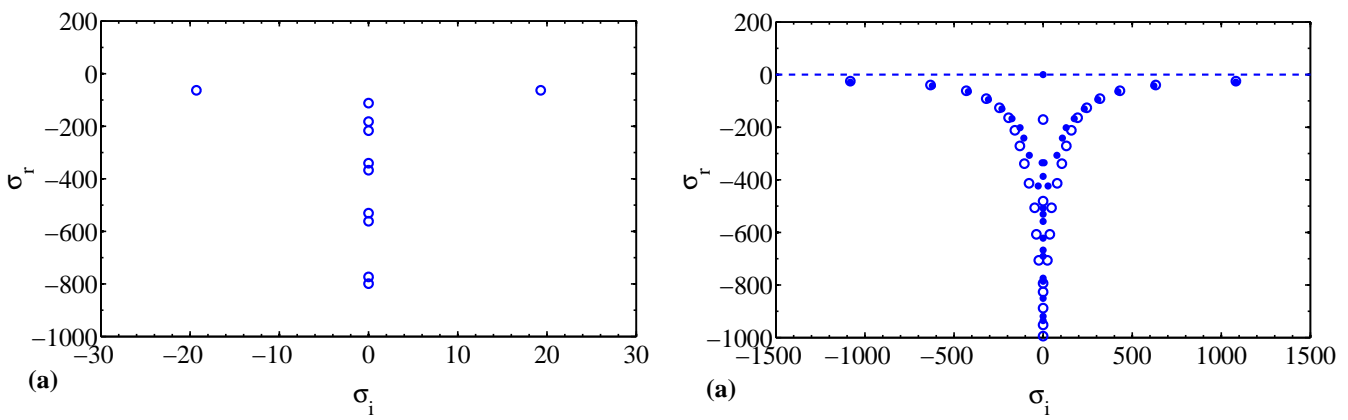

FigURE 7. Eigenspectra for a power-law fluid undergoing an axisymmetric perturbation $m=0$ with $k=\pi, n=0.5, \eta=0.881$, varying $R e_{2}$ (a) on the Rayleigh line $R e_{2}=454.71, R e_{1}=500$ (b) $R e_{2}=-4000,(\circ) R e_{1}=500,(\bullet) R e_{1}=R e_{1 c}=828.14$.
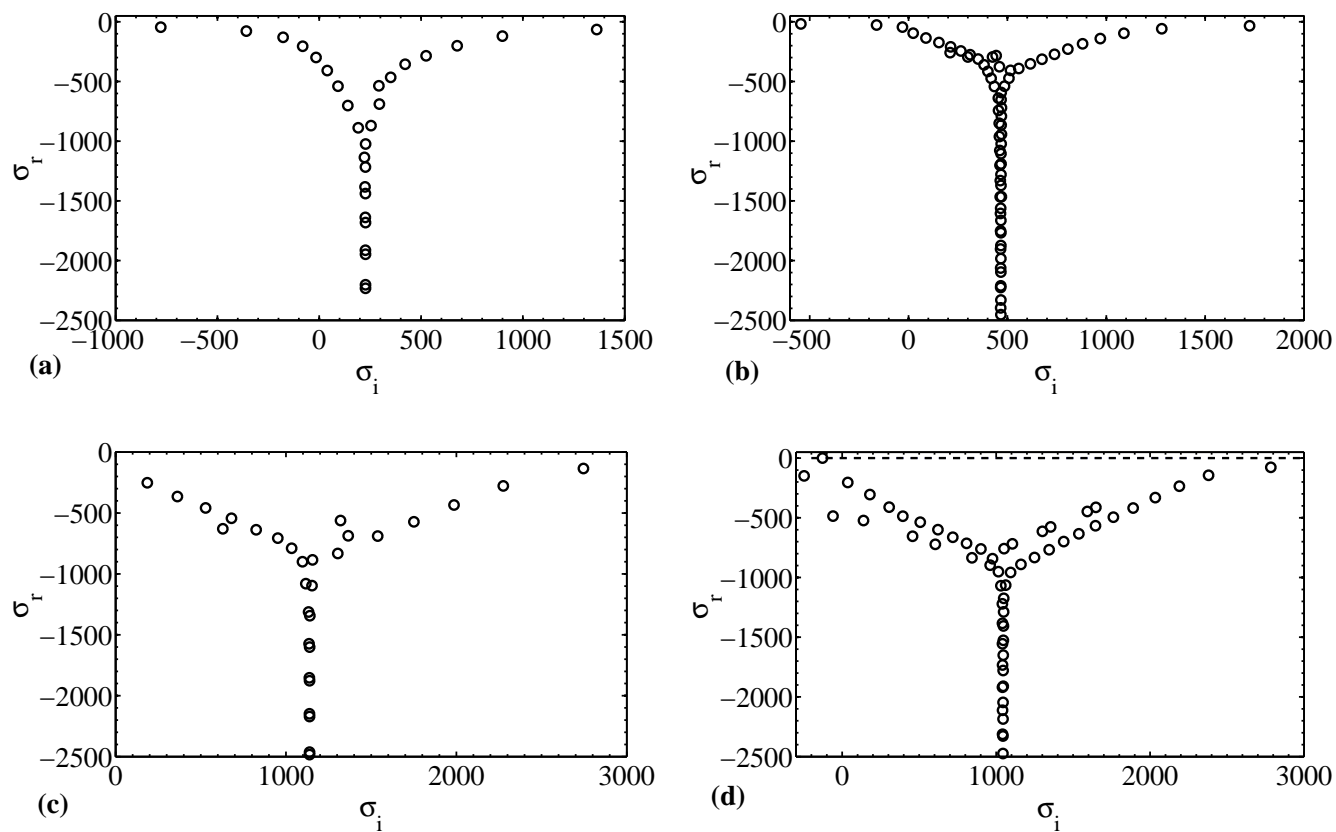

FIgURE 8. Eigenspectra for a power-law fluid undergoing a non axisymmetric perturbation with $n=0.5, \eta=0.881$. The outer Reynolds number and the axial wavenumber are fixed: $R e_{2}=-4000$ and $k=\pi$ respectively. (a) $m=1, R e_{1}=400$, (b) $m=2, R e_{1}=400$, (c) $m=5, R e_{1}=400,(\mathrm{~d}) m=5, R e_{1}=R e_{1 c}=878.55$.

stationary and consists of two counter-rotating vortices. Similar results are obtained for a Carreau fluid. 


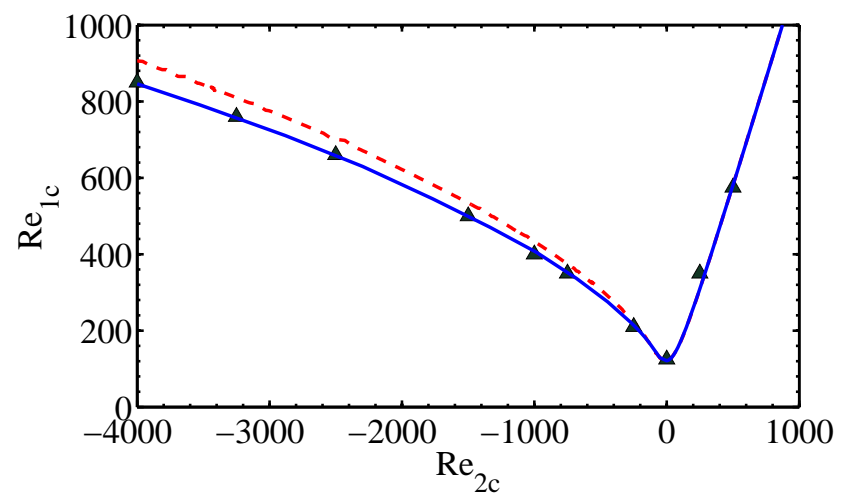

FiguRE 9. Marginal stability curves in the plane $\left(R e_{1}, R e_{2}\right)$ for a Newtonian fluid with $\eta=0.881$ : Comparison with Meseguer (2002). (Continuous line) our results for 3D perturbations, (dashed line) our results for axisymmetric perturbations $m=0,(\triangle)$ Meseguer (2002).

\subsubsection{Non-axisymmetric perturbation $m \neq 0$}

Numerical computations show no fundamental differences in the eigenspectra shape for Newtonian and shear-thinning fluids. In order not to overload the paper, we have chosen to present only the power-law results. The classification of the eigenspectra is less evident than in the axisymmetric case. According to Gebhart \& Grossmann (1993), the structure of the eigenspectra depends on the relative value of the axial wavenumber compared to the modified azimuthal wavenumber $m / r_{m}$ with $r_{m}=\left(R_{1}+R_{2}\right) / 2$. If $k \geqslant m / r_{m}$, the spectrum structure is close to that of an azimuthal invariant flow. On the contrary, if $k \leqslant m / r_{m}$, features of an axial invariant flow dominate. Figure 8 shows that with increasing $m$, the eigenvalue spectrum evolves from a "jet-like" structure (figure 8(a)) characteristic of axisymmetric perturbation flow to a " $Y$-shaped" spectrum characteristic of axial invariant perturbation (Gebhart \& Grossmann 1993). Figure 8(d) shows that when $R e_{1}$ is increased until marginal stability $\left(\sigma_{r}=0\right)$, the critical mode corresponds to time-dependent spiral vortices, with an angular phase velocity $\omega=-\sigma_{i} / \mathrm{m}$. 


\subsection{Marginal stability curves}

First of all, a validation test was performed by confronting our results to studies previously published in the literature. In figure 9 our results are compared to those obtained by Meseguer (2002) for 3D perturbations in a Newtonian fluid. A very good agreement is observed. In the case of counter-rotating cylinders, the critical $R e_{1 c}$ is lower for non axisymmetric modes $(m \neq 0)$ than for axisymmetric ones $(m=0)$, in agreement with the literature (Krueger et al. 1966; Langford et al. 1988). For power-law fluids, figure 10 shows the influence of the shear-thinning index on the critical conditions. Globally, for fixed $R e_{2}$, shear-thinning reduces $R e_{1 c}$ except in the neighborhood of $R e_{2}=0$ with a large gap and for co-rotating cylinders with small gaps, where $R e_{1 c}$ increases slightly. Similar evolutions are observed for Carreau fluids. For $\lambda \geqslant 10$, we recover the power-law results. The decrease of $R e_{1 c}$ with increasing shear-thinning effects can be analyzed using the energy equation obtained by multiplying the linearized momentum equations with the complex conjugate $\boldsymbol{u}^{*}$ and by integrating between the two cylinders

$$
\begin{gathered}
\mathcal{R} e(\sigma)\|\boldsymbol{u}\|^{2}=-R e_{1} \int_{R_{1}}^{R_{2}} f(r) r \mathrm{~d} r-\int_{R_{1}}^{R_{2}} g_{1}(r) r d r+\int_{R_{1}}^{R_{2}} g_{2}(r) r \mathrm{~d} r \quad \text { with } \\
f(r)=\dot{\gamma}_{r \theta}^{b} \frac{u^{*} v+u v^{*}}{2} \\
g_{1}(r)=\mu^{b}\left[\frac{1}{2}\left(\left|\dot{\gamma}_{r r}\right|^{2}+\left|\dot{\gamma}_{\theta \theta}\right|^{2}+\left|\dot{\gamma}_{z z}\right|^{2}\right)+\left|\dot{\gamma}_{r \theta}\right|^{2}+\left|\dot{\gamma}_{r z}\right|^{2}+\left|\dot{\gamma}_{\theta z}\right|^{2}\right] \\
g_{2}(r)=\left(\mu^{b}-\mu_{t}\right)\left|\dot{\gamma}_{r \theta}^{\prime}\right|^{2} .
\end{gathered}
$$

with $\|\boldsymbol{u}\|^{2}=\int_{R_{1}}^{R_{2}} \frac{1}{2}\left(u u^{*}+v v^{*}+w w^{*}\right) r \mathrm{~d} r$.

In equation (6.4), the first term in the right hand side is a production term. It is associated with the work of the Reynolds-stress, $-u v$, on the wall-normal basic shear $\dot{\gamma}_{r \theta}^{b}$. The second term represents viscous dissipation. The third term arises from the viscosity perturbation due to shear-thinning; it is positive, and can thus be regarded as a source of energy for the perturbation. Following Ranganathan \& Govindarajan (2001), it is convenient to 

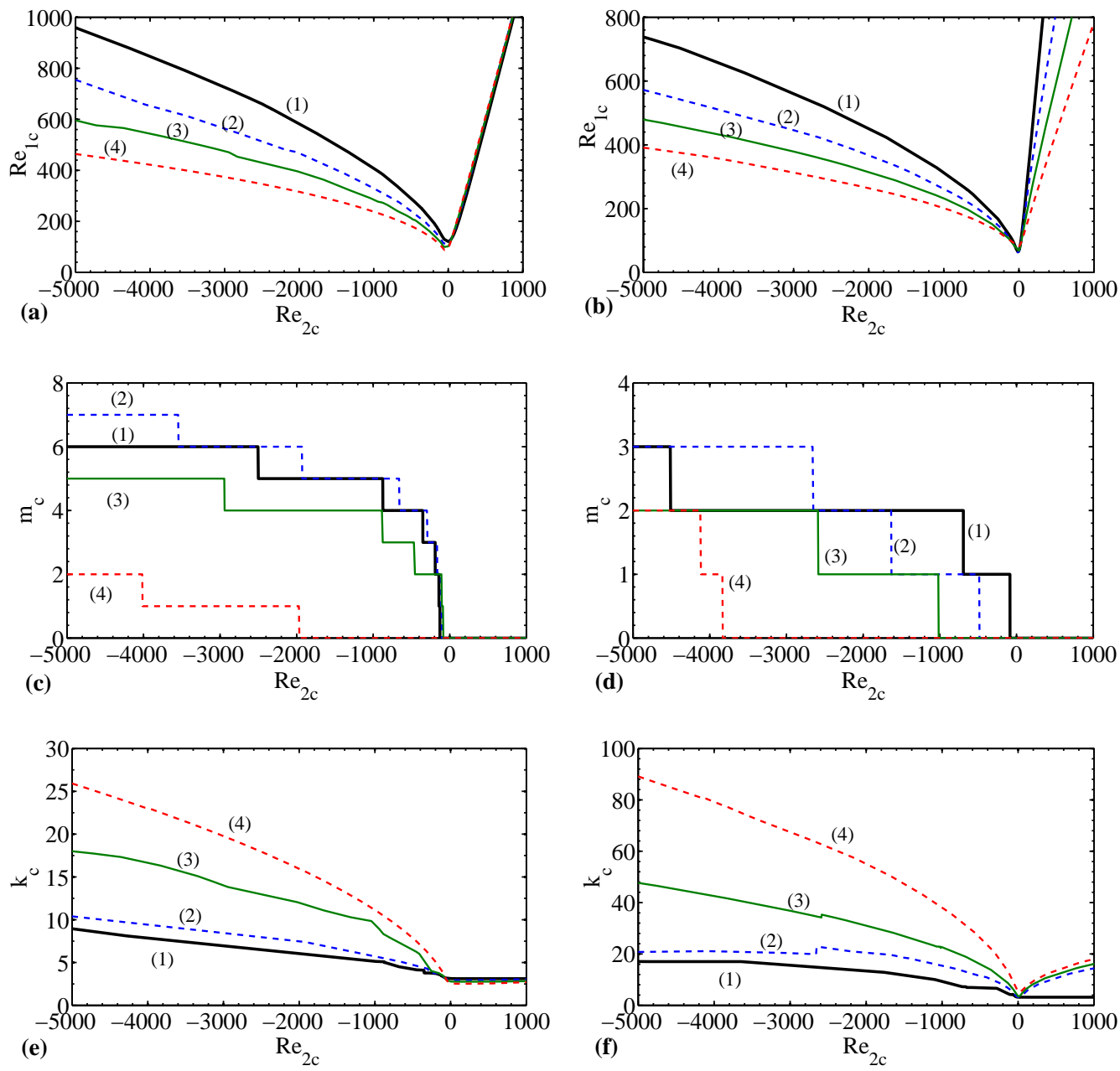

Figure 10. Critical conditions of instability for a power-law fluid with two radius ratios, $\eta=0.881$ (figures on the left) and $\eta=0.4$ (figures on the right). Effect of the shear thinning index. (a) and (b) Marginal stability curves in plane $\left(R e_{1}, R e_{2}\right),(\mathrm{c})$ and (d) critical azimuthal wavenumbers, (e) and (f) critical axial wavenumbers. (1) Newtonian fluid, $n=1$, (2) $n=0.7$, (3) $n=0.5$ and (4) $n=0.3$.

compute and compare the space-averaged production and dissipation terms $\Gamma^{+}$and $\Gamma^{-}$ respectively defined by

$$
\Gamma^{+}=\frac{R e_{1} \int_{R_{1}}^{R_{2}} f(r) r \mathrm{~d} r}{\|\boldsymbol{u}\|^{2}} \quad \text { and } \quad \Gamma^{-}=\frac{\int_{R_{1}}^{R_{2}} g_{1}(r) r d r-\int_{R_{1}}^{R_{2}} g_{2}(r) r \mathrm{~d} r}{\|\boldsymbol{u}\|^{2}}
$$



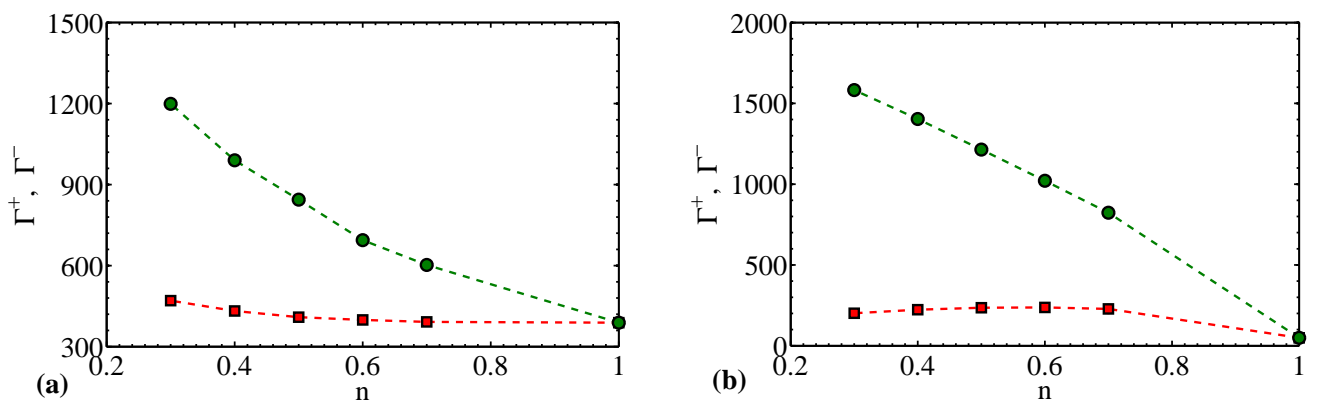

Figure 11. Space average of (o) the production term $\Gamma^{+}$and $(\square)$ the viscous dissipation term $\Gamma^{-}$defined by equation (6.8) for a power-law fluid with $\eta=0.4$ as a function of shear-thinning index, n. (a) $R e_{2}=-2025$, (b) $R e_{2}=500$.

At criticality, the production term is exactly balanced by the dissipation term, $\Gamma^{+}=\Gamma^{-}$, as shown in figure 11 in the case of a Newtonian fluid. The influence of shear-thinning effects on the energy budget can be appreciated by comparing the results obtained for a Newtonian fluid with those of a power-law at the same value of $R e_{1}$, i.e. $R e_{1}=$ $R e_{1 c}$ (Newtonian). The critical azimuthal and axial wavenumbers are evaluated for the parameters $R e_{1}=R e_{1 c}($ Newtonian$), R e_{2}$ and $n$. With increasing shear-thinning effects, the average viscous dissipation remains close to that of a Newtonian fluid, while the production term increases significantly, rendering the flow less stable compared to the Newtonian case. The main factor determining stability or instability of the flow is thus the exchange of energy between the base flow and the perturbation. In figure 12, the location of the maxima $f$ and $\left(g_{1}+g_{2}\right)$, functions defined in equations $(6.5-6.7)$ are plotted against $R e_{2}$. It appears that the maxima of the energy exchange and of viscous dissipation occur near the inner cylinder. The increase of $\Gamma^{+}$with increasing shear-thinning effects may be attributed to the increase of the inner velocity gradient. Concerning the critical azimuthal and axial wavenumbers, in the co-rotating regime, the critical mode is axisymmetric (figure 10(c)(d)). The primary bifurcation leads to time independent Taylor-vortex flow. In the case of counter-rotating regime, the critical azimuthal wavenumber increases with 


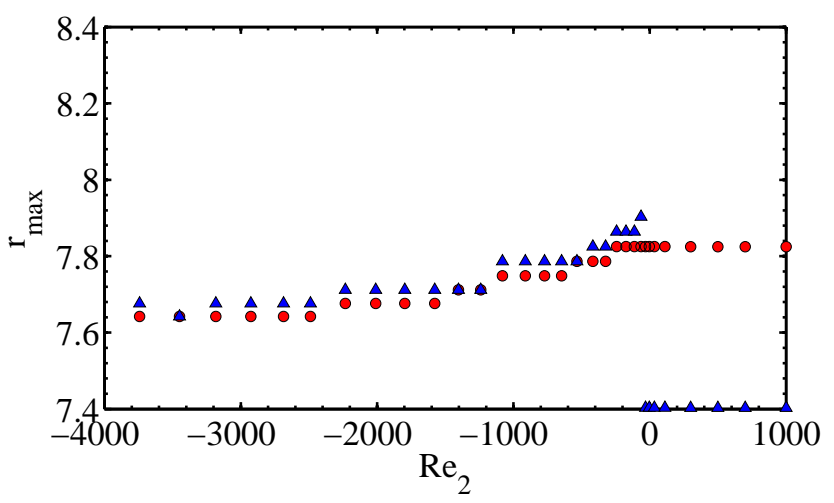

Figure 12. Radii at which functions $(\circ) f(r)$ and $(\triangle) g_{1}(r)+g_{2}(r)$ defined by equations (6.5) - (6.7), are maximal, for a power-law fluid with $n=0.5$ and $\eta=0.881$.

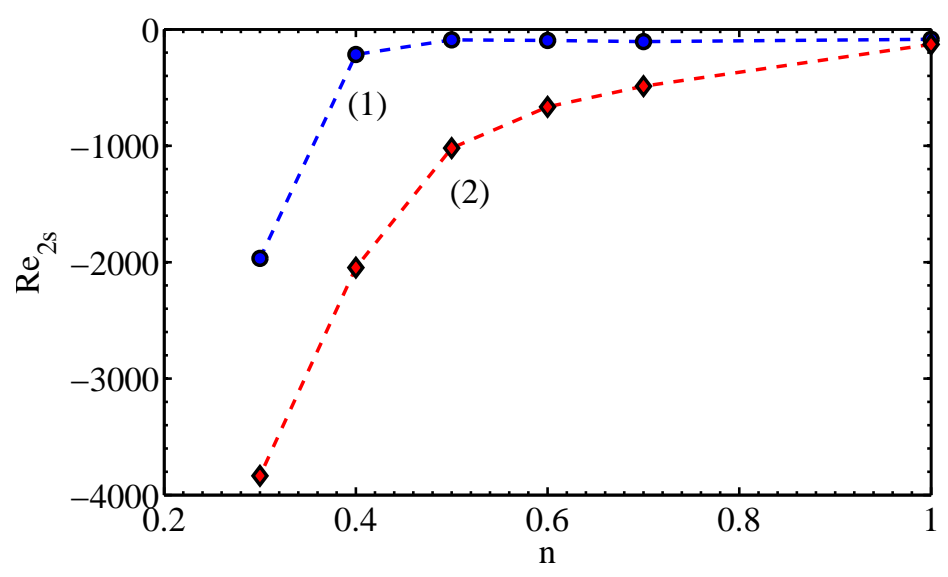

FIGURE 13. Power-law fluids. Influence of the shear-thinning index on the critical outer Reynolds number $R e_{2 s}$ at which the transition to spirals takes place for (1) $\eta=0.881$ and (2) $\eta=0.4$. $\left|R e_{2}\right|$. There is a limit value of $R e_{2}$, say $R e_{2 s}$ under which the primary bifurcation leads to axisymmetric toroidal structures $\left(m_{c}=0\right)$ and over which the bifurcation leads to spiral vortices $\left(m_{c} \geqslant 1\right)$. Spiral vortices are traveling waves in axial and azimuthal directions. Figure 13 shows the variation of $R e_{2 s}$ with shear-thinning index for both a wide and a thin gap. For strong shear-thinning effects, the critical mode remains axisymmetric for large negative $R e_{2}$. The axial wavelength decreases with increasing negative $R e_{2}$ and increasing shear-thinning effects. This may be attributed to the decrease of the "effective 
gap" where the Rayleigh instability crietrion holds, i.e. the gap between the inner cylinder and the "nodal surface" where $V^{b}=0$.

\section{Short-time behavior: transient growth and optimal perturbation}

The traditional approach of stability based on eigenvalues is not sufficient to describe the temporal behavior of the disturbance at all times because of the non-normality of the linear stability operator. The non-normality of the linear stability operator $\mathscr{L}=\mathcal{M}^{-1} \mathcal{L}$ is characterized by using the $\varepsilon$-pseudospectrum tools (see for instance Trefethen et al. (1993); Reddy et al. (1993), and references therein). More details are given in appendix E. This mathematical property means that there is a potential for energy extraction from the base flow by a subspace of perturbations leading to transient growth, despite the absence of exponential instability.

\subsection{Transient growth and characteristics of optimal perturbation}

The transient evolution of a perturbation kinetic energy in the linear regime is determined by following the methodology described by Schmid \& Henningson (2001). It is presented here only for the $(\tilde{u}, \tilde{v})$ formulation, but similar development applies to the $(\tilde{u}, \tilde{w})$ formulation. For a given Fourier mode, the disturbance instantaneous kinetic energy is given by

$\|\boldsymbol{q}(t)\|_{E}^{2}=\int_{R_{1}}^{R_{2}}\left[|\tilde{u}|^{2}+\frac{1}{k^{2}} \frac{1}{r^{2}}\left|\frac{d}{d r}(r \tilde{u})\right|^{2}+\left(\frac{m^{2}}{r^{2} k^{2}}+1\right)|\tilde{v}|^{2}+\frac{i m}{k r}\left(\frac{\tilde{v}}{r} \frac{d}{d r}\left(r \tilde{u}^{*}\right)-\frac{\tilde{v}^{*}}{r} \frac{d}{d r}(r \tilde{u})\right)\right] r d r$

which is a function of time and of the initial condition, $\boldsymbol{q}_{0}=\left(\tilde{u}_{0}, \tilde{v}_{0}\right)^{T}=\boldsymbol{q}(r, t=0, m, k)$. The maximum kinetic energy amplification at time $t$ over all initial conditions is defined by

$$
G(t, m, k)=\sup _{\boldsymbol{q}_{0} \neq 0} \frac{\|\boldsymbol{q}(t)\|_{E}^{2}}{\|\boldsymbol{q}(0)\|_{E}^{2}}
$$


The maximum energy transient growth over all times is

$$
G^{\max }(m, k)=\sup _{t \geqslant 0} G(t, m, k) .
$$

The maximum of $G^{\max }$ over all pairs $(m, k)$ is denoted $G^{o p t}$ and is reached by the optimal perturbation at time $t^{o p t}$.

The quantity $G(t)$ is obtained as follows. Consider the linear subspace $\mathcal{S}_{M}$ spanned by the $M$ eigenfunctions $\boldsymbol{q}_{j}=\left(\tilde{u}_{j}, \tilde{v}_{j}\right)$ corresponding to the first $M$ eigenvalues $\sigma_{j}$ of the spectrum of $\mathscr{L}$, sorted in order of decreasing growth rate. Any admissible perturbation can be written as a linear combination of $\boldsymbol{q}_{j}$,

$$
\boldsymbol{q}=\sum_{j=1}^{j=M} K_{j} \boldsymbol{q}_{j}=\boldsymbol{Q} \cdot \boldsymbol{K}
$$

where $\boldsymbol{Q}$ is the eigenvector matrix. One can approximate $G(t)$ by computing the maximum possible energy amplification at time $t$ over all possible initial combinations of the $M$ eigenfunctions.

The initial value problem (5.12) can be expressed as

$$
\boldsymbol{K}=e^{\boldsymbol{\Sigma} t} \boldsymbol{K}_{0}
$$

with $\boldsymbol{\Sigma}=\operatorname{diag}\left(\sigma_{1}, \ldots, \sigma_{M}\right)$ the diagonal matrix of eigenvalues and $\boldsymbol{K}_{0}$ the vector of expansion coefficients that defines the initial perturbation. The energy norm of $\boldsymbol{q}(t)$ is

$$
\|\boldsymbol{q}(t)\|_{E}^{2}=\boldsymbol{K}^{*} \boldsymbol{M} \boldsymbol{K}=\boldsymbol{K}^{*} \boldsymbol{F}^{H} \boldsymbol{F} \boldsymbol{K}=\|\boldsymbol{F} \boldsymbol{K}\|_{2}^{2}
$$

where the superscript $H$ stands for the complex conjugate transpose, $\boldsymbol{M}$ is a positive definite Hermitian matrix, $\boldsymbol{M}=\boldsymbol{F}^{H} \boldsymbol{F}$, whose elements are obtained from the inner product of the $M$ eigenfunctions, associated to the energy norm (7.1). Hence,

$$
G(t)=\sup _{\boldsymbol{K}_{0} \neq 0} \frac{\|\boldsymbol{F} \boldsymbol{K}(t)\|_{2}^{2}}{\left\|\boldsymbol{F} \boldsymbol{K}_{0}\right\|_{2}^{2}}=\sup _{\boldsymbol{K}_{0} \neq 0} \frac{\left\|\boldsymbol{F} e^{\boldsymbol{\Sigma}_{t}} \boldsymbol{F}^{-1} \boldsymbol{F} \boldsymbol{K}_{0}\right\|_{2}^{2}}{\left\|\boldsymbol{F} \boldsymbol{K}_{0}\right\|_{2}^{2}}
$$



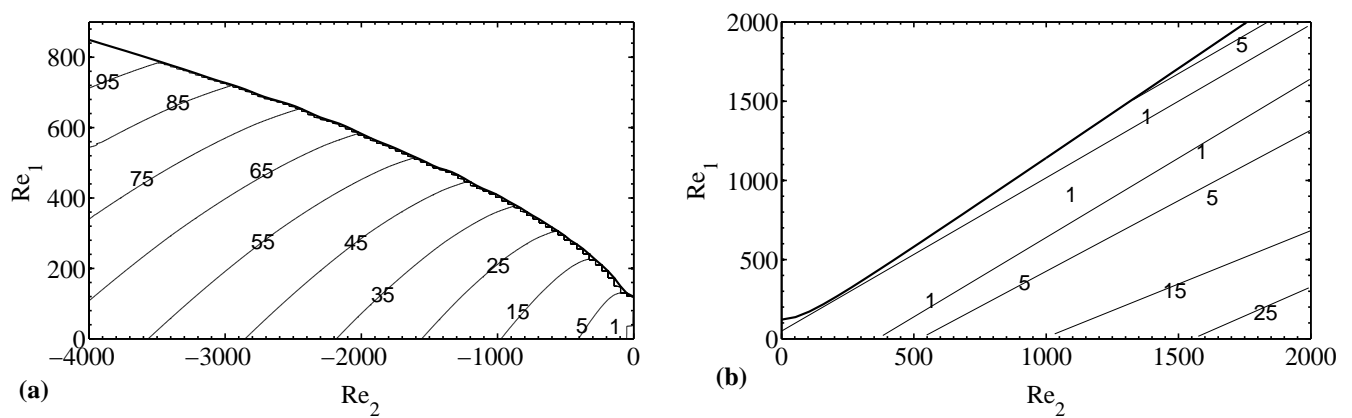

Figure 14. Contours of optimal energy growth $G^{o p t}$ in the plane $\left(R e_{1}, R e_{2}\right)$ for a Newtonian fluid with $\eta=0.881$ : (a) counter-rotating cylinders, (b) co-rotating cylinders. The thick line is the marginal stability curve.

As $\boldsymbol{F} \boldsymbol{K}_{0}$ is a vector, the energy growth can be expressed using the matrix 2-norm as

$$
G(t)=\left\|e^{\boldsymbol{\Sigma} t}\right\|_{E}^{2}=\left\|\boldsymbol{F} e^{\boldsymbol{\Sigma} t} \boldsymbol{F}^{-1}\right\|_{2}^{2}=s_{1}^{2}
$$

where $s_{1}$ is the largest singular value of the matrix $\boldsymbol{F} e^{\boldsymbol{\Sigma}_{t}} \boldsymbol{F}^{-1}$. Since the eigenfunctions have been expressed in terms of Chebyshev polynomials, all the calculations are recasted in terms of Chebyshev expansion coefficients. The number of collocation points needed for convergence to be achieved depends on the shear-thinning degree and on the magnitude of the Reynolds numbers. Globally, a resolution of $N=40$ is sufficient. However, for very strong shear-thinning effects say $n \leqslant 0.3$ in the case of the power-law model and $n \leqslant 0.3$ with $\lambda \geqslant 10$ for the Carreau model, the number of collocation points necessary to achieve convergence is increased up to $N=150$.

To begin with, we performed validation tests for a Newtonian fluid. In table 1, our results are compared to those of Meseguer (2002) and Maretzke et al. (2014). Very good agreement is observed. The conditions of Hristova et al. (2002) given in their table II were also simulated. A relative discrepancy of less than $1 \%$ was obtained.

As a reference case, we further studied the transient growth for a Newtonian fluid. Figure 14 shows contours of the optimal growth for a Newtonian fluid in the plane $\left(R e_{1}, R e_{2}\right)$. 


$\begin{array}{llcccccccccc}R e_{1} & R_{2} & k^{\text {opt }} & m^{\text {opt }} & G^{\text {opt }} & k^{\text {opt }} & m^{\text {opt }} & G^{\text {opt }} & k^{\text {opt }} & m^{\text {opt }} & G^{\text {opt }} \\ 591 & -2588 & 1.994 & 10 & 71.36 & 1.997 & 10 & 71.58 & 2.015 & 10 & 71.58 \\ 523 & -2975 & 1.996 & 11 & 71.58 & 1.998 & 11 & 71.81 & 1.985 & 11 & 71.80 \\ 473 & -3213 & 1.920 & 11 & 71.64 & 1.922 & 11 & 71.87 & 1.924 & 11 & 71.87 \\ 405 & -3510 & 1.839 & 11 & 71.75 & 1.841 & 11 & 71.99 & 1.833 & 11 & 71.99\end{array}$

TABLE 1. Comparison of the present results with those of Meseguer (2002) and Maretzke et al. (2014) at the same Reynolds numbers and $\eta=0.881$.
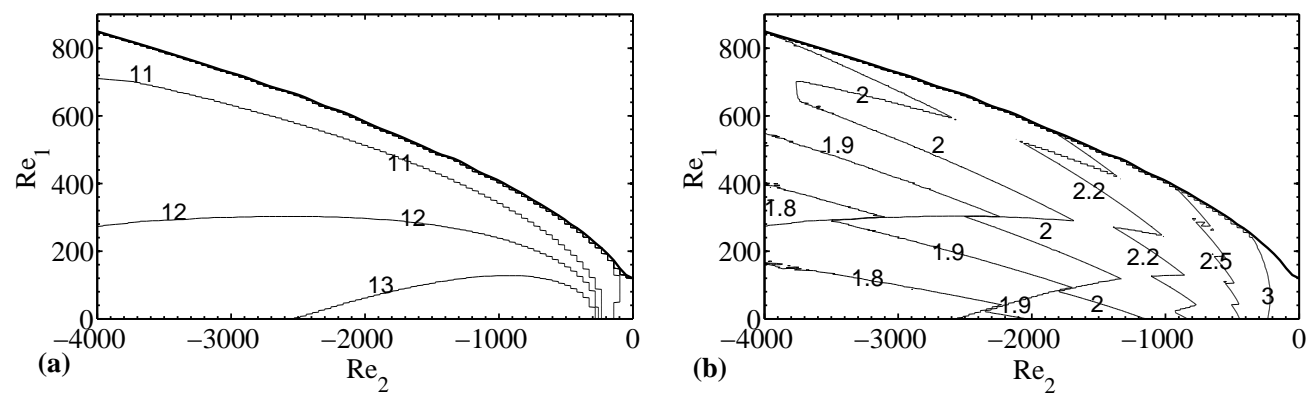

Figure 15. Contours of optimal wavenumbers in the $\left(R e_{1}, R e_{2}\right)$ plane for a Newtonian fluid flowing between counter-rotating cylinders with $\eta=0.881$. (a) Optimal azimuthal wavenumber $m^{\text {opt }}$ and (b) optimal axial wavenumber $k^{o p t}$. The thick line is the marginal stability curve.
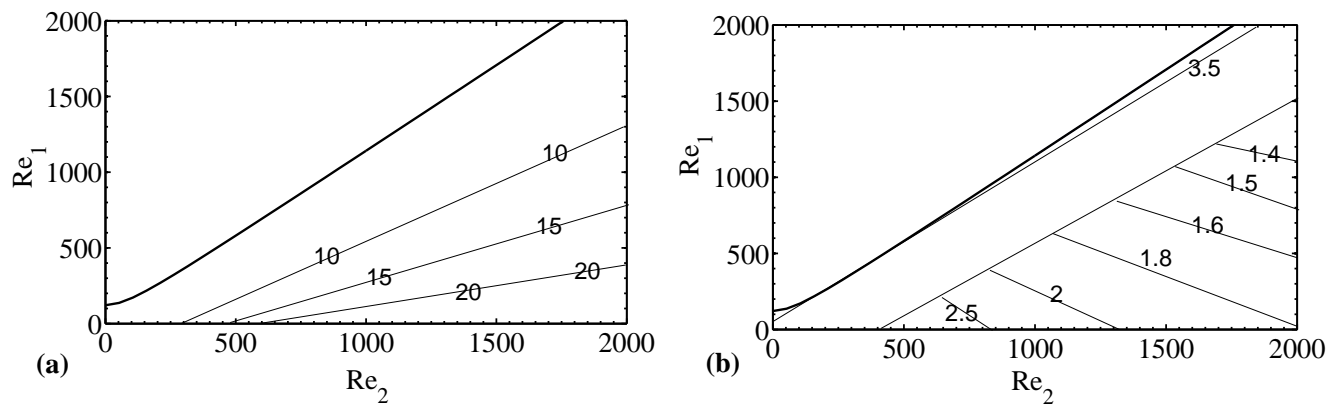

Figure 16. Contours of optimal wavenumbers in the $\left(R e_{1}, R e_{2}\right)$ plane for a Newtonian fluid flowing between co-rotating cylinders with $\eta=0.881$. (a) Optimal azimuthal wavenumber $m^{o p t}$ and (b) optimal axial wavenumber $k^{\text {opt }}$. The thick line is the marginal stability curve. 

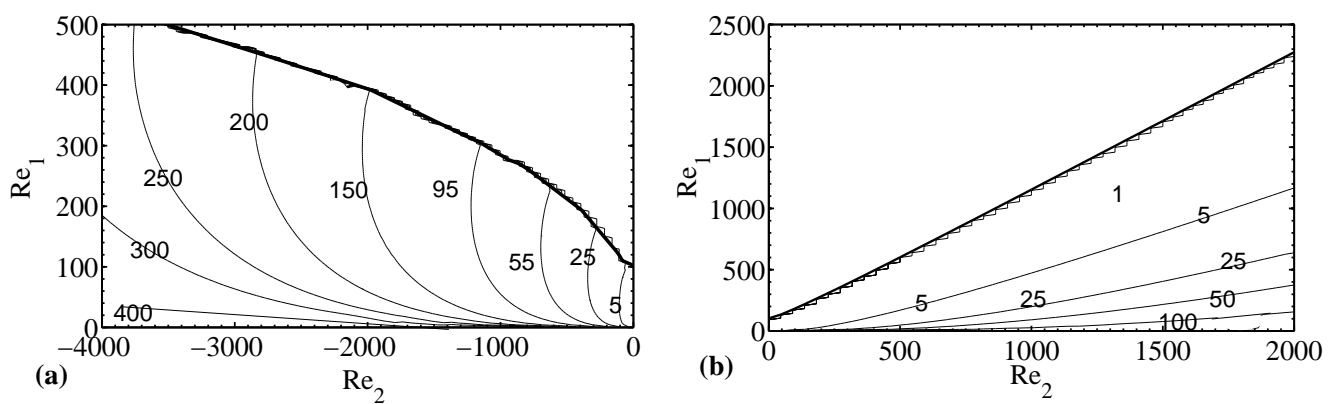

Figure 17. Contours of optimal energy growth $G^{o p t}$ in the plane $\left(R e_{1}, R e_{2}\right)$ for a power-law fluid with $n=0.5$ and $\eta=0.881$ : (a) counter-rotating cylinders, (b) co-rotating cylinders. The thick line is the marginal stability curve.

The corresponding axial and azimuthal wavenumbers are represented in figures 15 and 16. It is interesting to observe that in the counter-rotating regime, the optimal azimuthal wavenumber varies little $\left(10<m^{o p t}<13\right)$ and is much higher than the critical wavenumber $m_{c}$. Concerning $k^{\text {opt }}$, it is smaller than the critical axial wavenumber $k_{c}$. For a shearthinning fluid, the contours of optimal energy amplification are displayed in figure 17 . The amplification appears to be much larger than for a Newtonian fluid. In table 2, the optimal growth conditions $G^{o p t}, k^{o p t}, m^{o p t}$ and $t^{o p t}$ are compared for Newtonian, powerlaw and Carreau fluids at given $R e_{1}$ and $R e_{2}$ for thin and wide gaps. It is observed that the optimal amplification is highest for Carreau fluid and at thin gap. The azimuthal and axial optimal wavenumbers are practically similar for Newtonian and shear-thinning fluids. The optimal time is greater for power-law and even greater for Carreau fluids.

These results may be presented in a concise manner by using the shear Reynolds number $R e$, and the rotation number $R_{\Omega}$. This representation is more relevant in the determination of scaling laws for the optimal energy amplification (Maretzke et al. 2014). Figure 18 shows the characteristics of the optimal perturbation as a function of $R e$ for different values of $R_{\Omega}$. We recover the scaling $G^{o p t} \propto R e^{2 / 3}$ for sufficiently large $R e$ as indicated by Maretzke et al. (2014). The optimal azimuthal wavenumber varies slightly with $R_{\Omega}$. At 


$$
R e_{1} \quad R_{2} \quad k^{o p t} m^{o p t} \quad G^{o p t} \quad t^{o p t}
$$

\begin{tabular}{|c|c|c|c|c|c|c|c|}
\hline \multirow{3}{*}{$\eta=0.881$} & Newtonian $(n=1)$ & 101 & -1061.2 & 2.176 & 13 & 20.62 & $9.3810^{-3}$ \\
\hline & Power-law $(n=0.5)$ & 101 & -1061.2 & 1.647 & 11 & 88.16 & $1.7110^{-2}$ \\
\hline & Carreau $(n=0.5 ; \lambda=10)$ & 101 & -1061.2 & 1.736 & 11 & 231.25 & $2.4110^{-2}$ \\
\hline \multirow{3}{*}{$\eta=0.4$} & Newtonian $(n=1)$ & 101 & -1061.2 & 1.136 & 3 & 4.288 & $6.9710^{-3}$ \\
\hline & Power-law $(n=0.5)$ & 101 & -1061.2 & 0.911 & 2 & 12.59 & $1.1510^{-2}$ \\
\hline & Carreau $(n=0.5 ; \lambda=10)$ & 101 & -1061.2 & 1.221 & 2 & 37.17 & $1.4410^{-2}$ \\
\hline
\end{tabular}

TABLE 2. Comparison of the optimal energy growth at $\eta=0.881$ and $\eta=0.4$ for the different fluid models considered
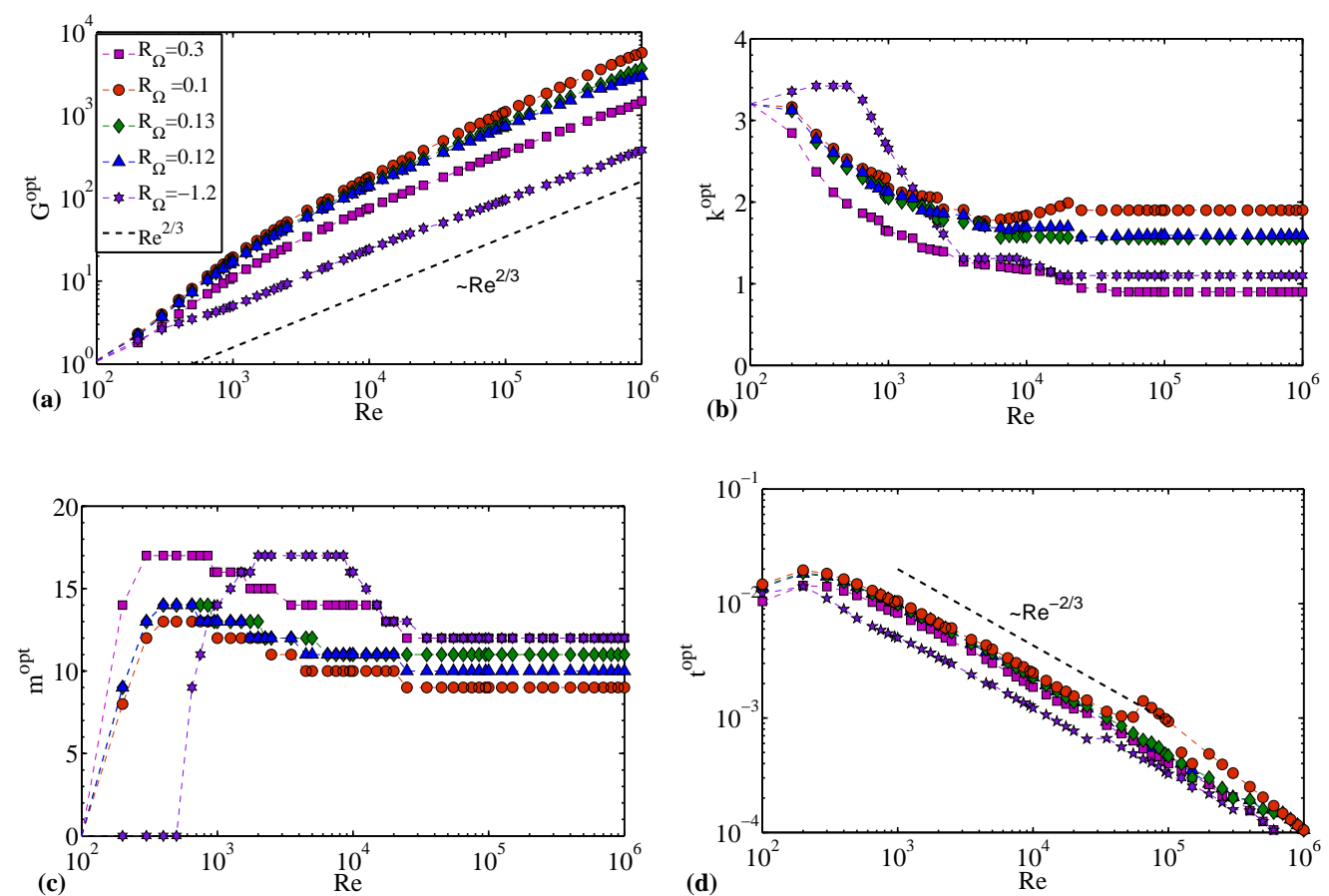

FIGURE 18. Optimal energy growth and characteristics of the optimal perturbation as a function of the shear Reynolds number $R e$ for different values of the rotation number $R_{\Omega}$. Newtonian fluid with $\eta=0.881$. (a) Optimal energy growth $G^{\text {opt }}$, (b) optimal axial wavenumber $k^{\text {opt }}$, (c) optimal azimuthal wavenumber $m^{o p t}$ and (d) optimal time $t^{o p t}$. 

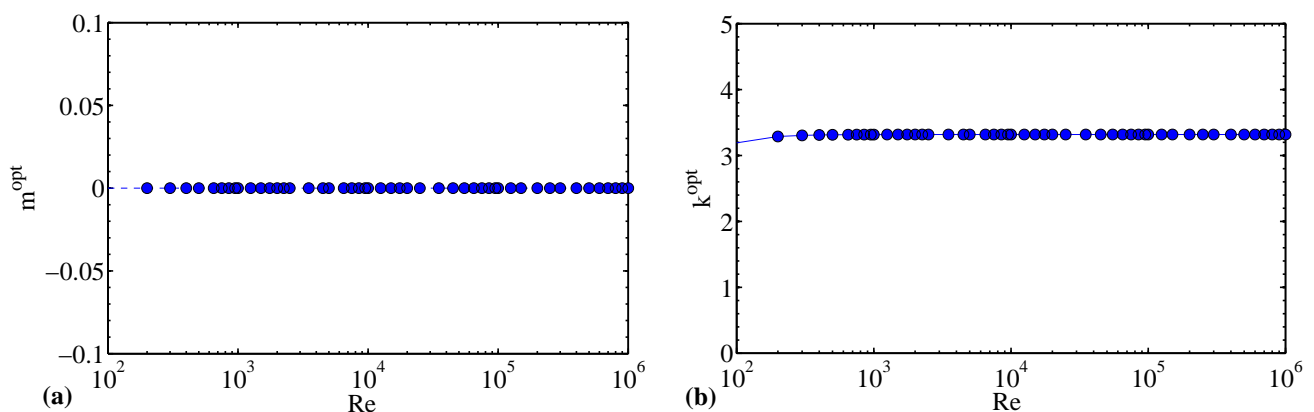

FIGURE 19. Structure of the optimal perturbation along the Rayleigh line $\left(R_{\Omega}=-1\right)$ for a Newtonian fluid and $\eta=0.881$. (a) Azimuthal and (b) axial wavenumbers as a function of the shear Reynolds number.
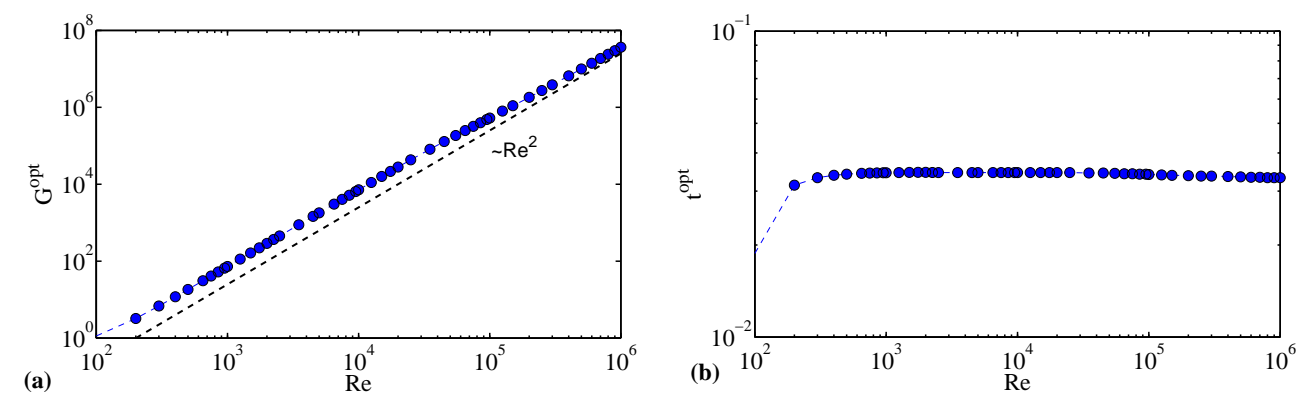

FiguRE 20. (a) Optimal energy growth and (b) optimal time versus shear Reynolds number along the Rayleigh line $\left(R_{\Omega}=-1\right)$ for a Newtonian fluid with $\eta=0.881$.

$\eta=0.881$, figure $18(\mathrm{c})$ shows again that asymptotically $10 \leqslant m^{\text {opt }} \leqslant 13$. In this range of $m^{\text {opt }}$, the kinetic energy amplification does not change significantly. Concerning the axial wavenumber, it is observed that it is much lower than the linear critical wavenumber $k_{c}$, figure 18(b). The optimal time $t^{o p t}$ decreases asymptotically as $t^{\text {opt }} \propto R e^{-2 / 3}$.

For the particular case of $R_{\Omega}=-1$, i.e. along the Rayleigh line, the optimal perturbation is axisymmetric with $k^{o p t} \approx \pi$ as shown by figure 19 . The optimal amplification varies as, $G^{o p t} \propto R e^{2}$ (figure 20). The optimal time at which $G^{\text {opt }}$ is reached decreases very slightly with $R e$.

The characteristics of optimal perturbations were also determined for power-law and Carreau fluids as shown in figures 21 and 22. It is interesting to note that we recover the 

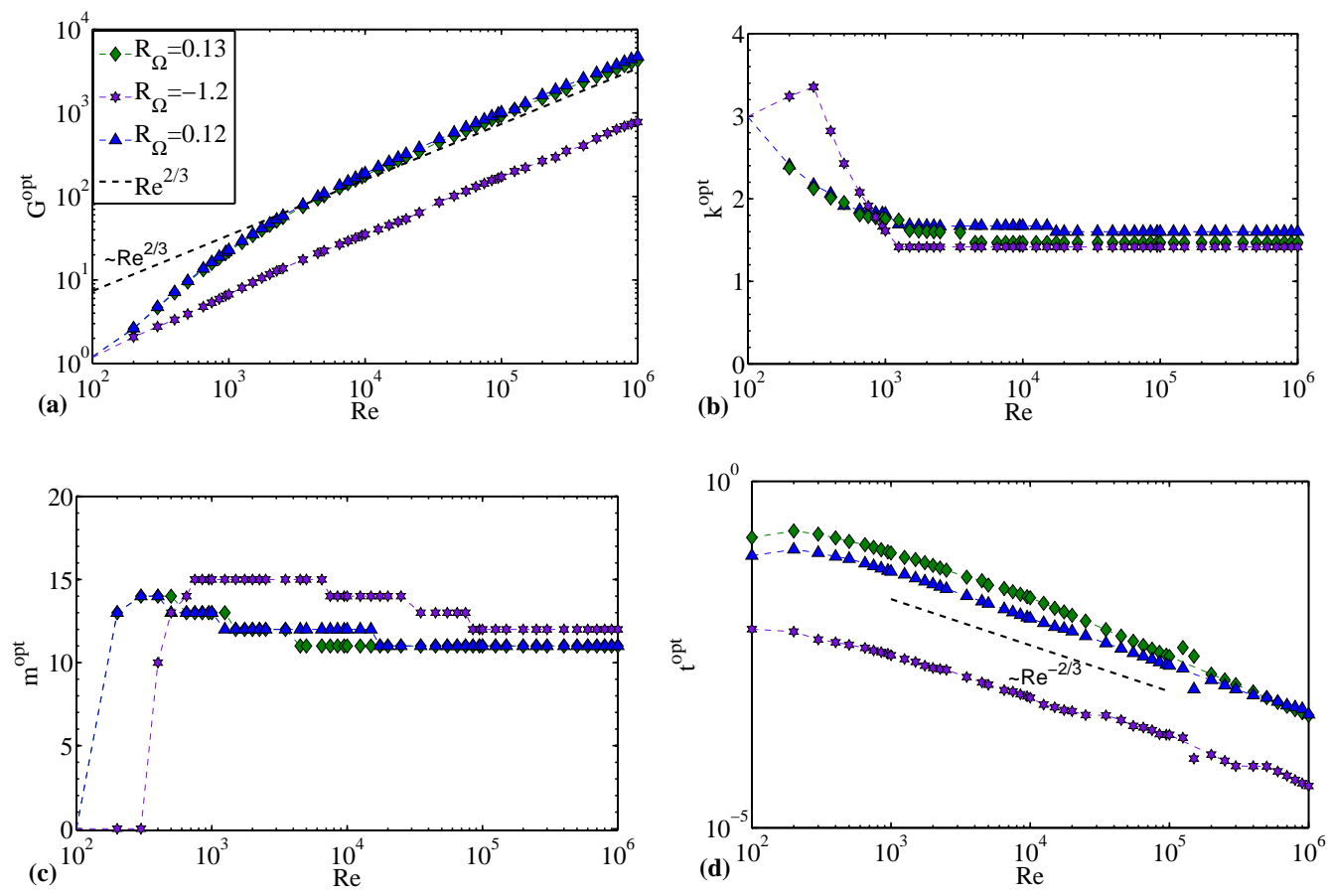

FiguRE 21. Optimal energy growth and characteristics of the optimal perturbation as a function of the shear Reynolds number $R e$ for different values of the rotation number $R_{\Omega}$. Power-law fluid with $n=0.5$ and $\eta=0.881$. (a) Optimal energy growth $G^{o p t}$, (b) optimal axial wavenumber $k^{o p t}$, (c) optimal azimuthal wavenumber $m^{\text {opt }}$ and (d) optimal time $t^{o p t}$.

same scaling as for a Newtonian fluid. Indeed, at large $R e, G^{o p t} \propto R e^{2 / 3} ; t^{o p t} \propto R e^{-2 / 3}$; $10 \leqslant m^{o p t} \leqslant 13$ and $1 \leqslant k^{o p t}<2$ much lower than $k_{c}$. Comparison with the Newtonian case indicates that for given $R e$ and $R_{\Omega}, G^{\text {opt }}$ increases with increasing shear-thinning degree as shown in figure 23. This is related to the increase of the non-normality of the linear operator emphasized in $\S ? ?$. The approximate scaling is found:

$$
\frac{G^{o p t}}{G_{n=1}^{o p t}} \propto \exp \left(-n^{\alpha}\right)
$$

where $G_{n=1}^{o p t}$ is the optimal energy growth for a Newtonian fluid and $\alpha=1 / 3$. 

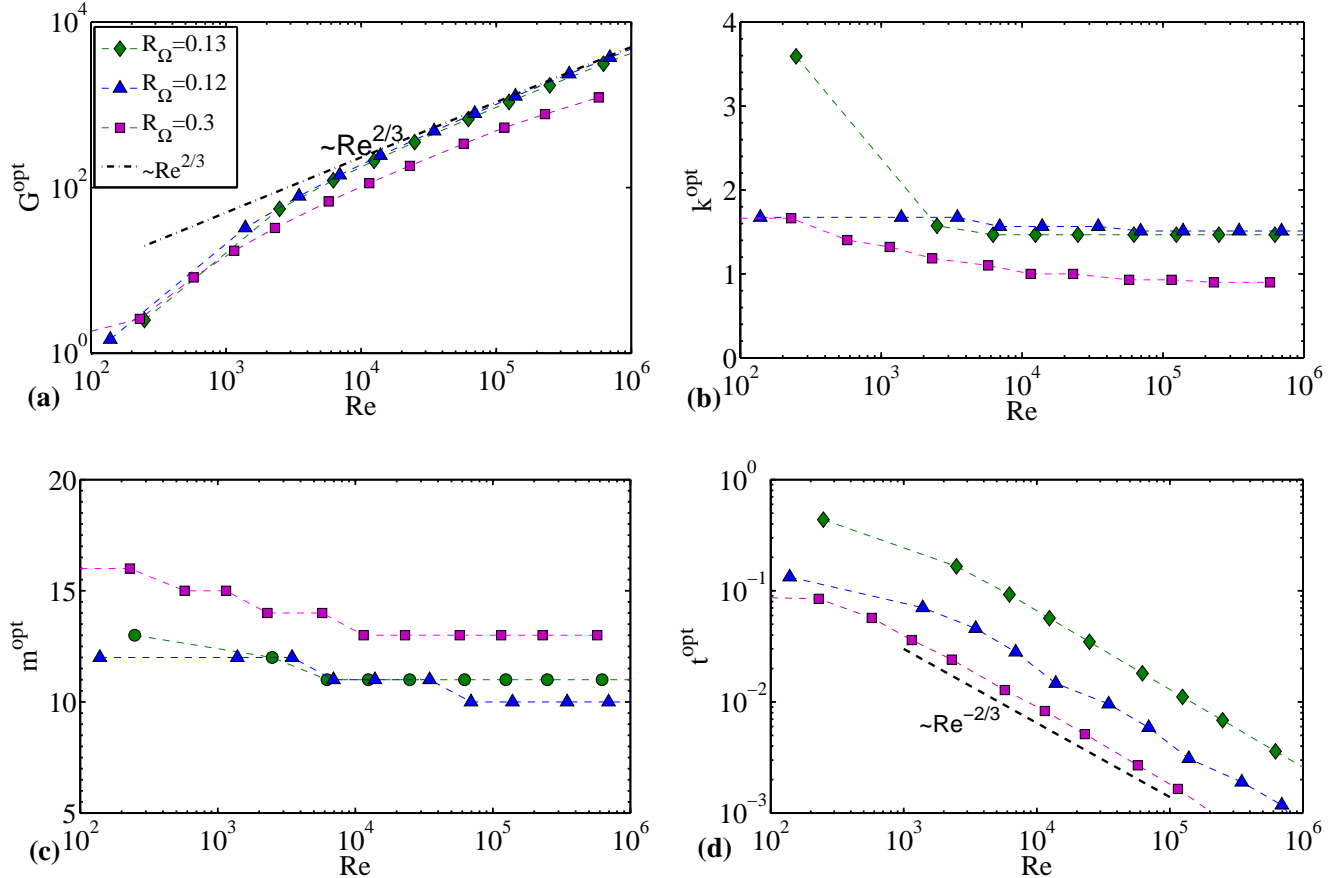

FIGURE 22. Optimal energy growth and characteristics of the optimal perturbation as a function of the shear Reynolds number for different values of the rotation number $R_{\Omega}$. Carreau fluid with $n=0.5, \lambda=10$ and $\eta=0.881$. (a) Optimal energy growth $G^{\text {opt }}$, (b) optimal axial wavenumber $k^{o p t}$, (c) optimal azimuthal wavenumber $m^{o p t}$ and (d) optimal time $t^{o p t}$.
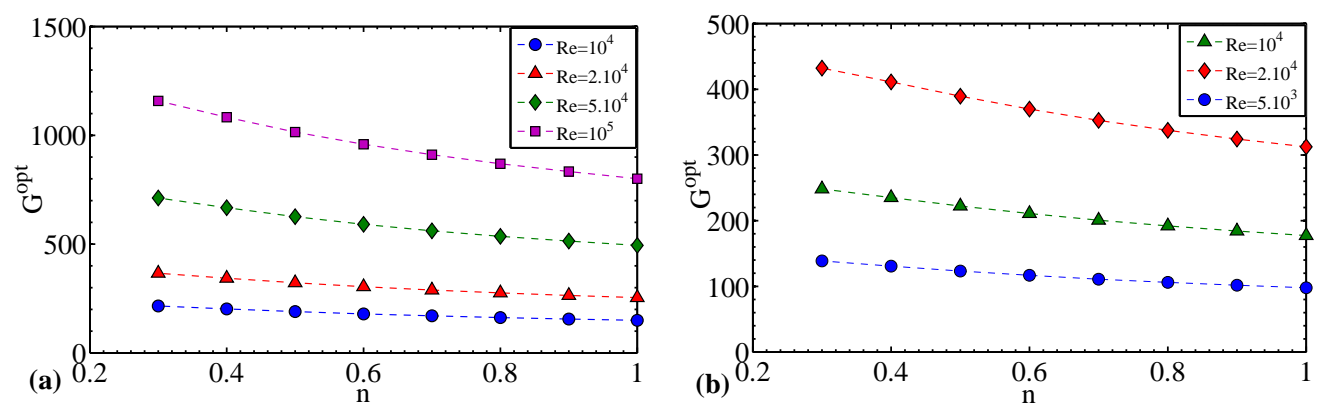

FigURE 23. Shear-thinning effect on the optimal energy growth for a power-law fluid with $\eta=0.881$, at different shear Reynolds numbers and fixed $R_{\Omega}$. (a) $R_{\Omega}=0.12$ and (b) $R_{\Omega}=0.1$.

\subsection{Evolution of the optimal perturbation: transient growth mechanism}

In this section, the optimal perturbation and its time evolution are described for different values of $R e_{1}$ and $R e_{2}$ in the linearly stable domain. 

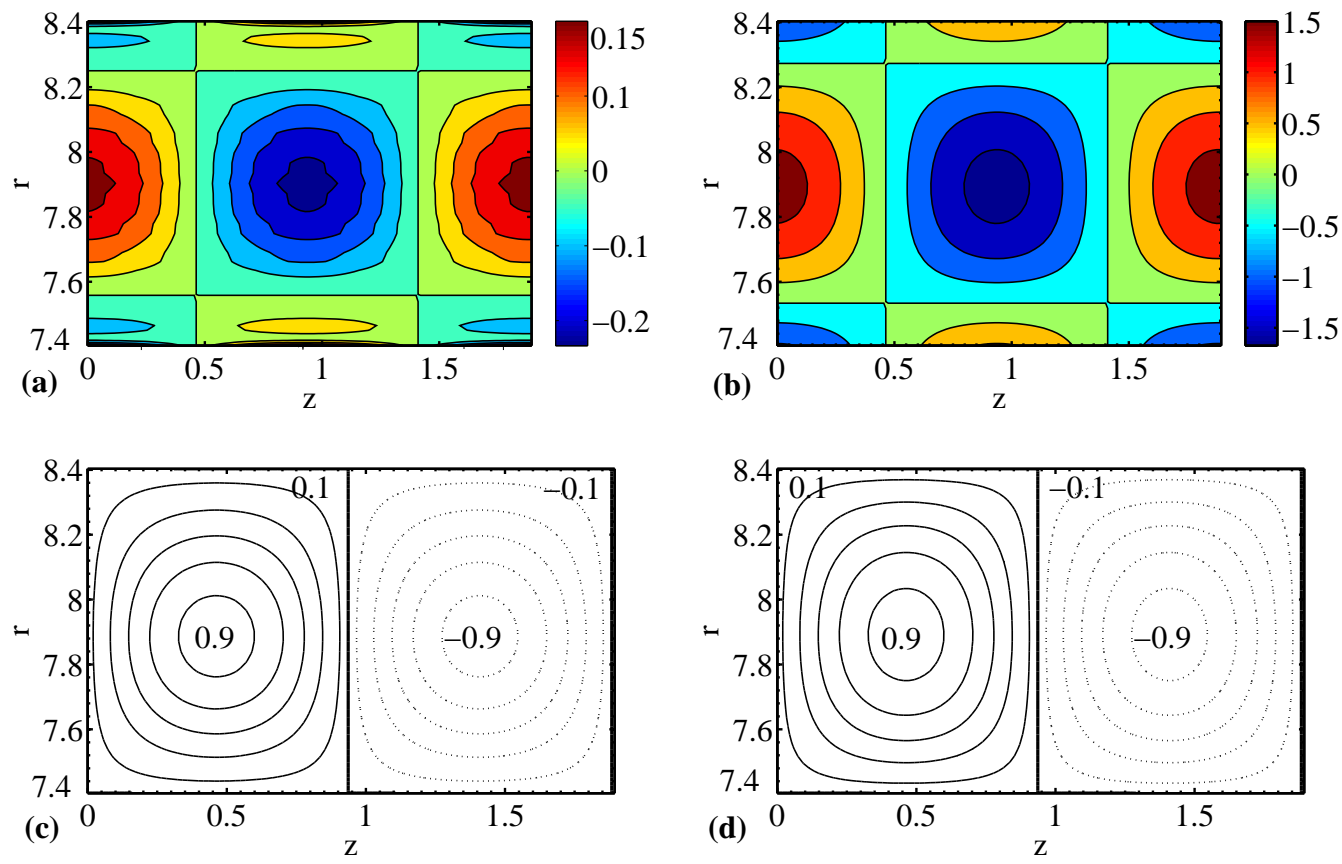

Figure 24. Optimal perturbation represented in the plane $(r, z)$ for a Newtonian fluid along the Rayleigh line, $\eta=0.881, R e_{1}=1135$ and $R e_{2}=1000$, with $m^{o p t}=0, k^{o p t}=3.32$ and $G^{o p t}=5.58$. Contours of the azimuthal vorticity component at $(a) t=0$ and $(b)$ optimal time $t=t^{o p t}$. Contours of the azimuthal velocity component at $(c) t=0, v_{\max }=0.52$ and $(d)$ optimal time $t=t^{o p t}, v_{\max }=0.1$; the contours are normalized by the maximum velocity at time $t$ and shown by steps of 0.2 . The solid lines represent positive values and the dotted lines represent negative values.

\subsubsection{Newtonian fluids}

For Newtonian fluids, numerical results show that the Rayleigh line should be distinguished from the rest of the domain. The contours of the azimuthal vorticity and azimuthal velocity of the optimal perturbation at initial time $t=0$ and optimal time $t=t^{o p t}$ are presented in figure 24 . It appears that the optimal perturbation is a superposition along the axial direction of high and low azimuthal velocity streaks. Figure 25 shows the contours of the local kinetic energy associated to the azimuthal, radial and axial components of the velocity perturbation. At initial time more than $99 \%$ of the kinetic 
energy of the perturbation is concentrated in the azimuthal component. At optimal time $t=t^{o p t}$, the kinetic energy is merely concentrated in the radial and axial components. This is also illustrated by figure 26 , where we have represented the spatial average of the kinetic energy contained in a volume $\mathcal{V}=2 \pi^{2}\left(R_{2}^{2}-R_{1}^{2}\right) / k$,

$$
E_{r}=\frac{1}{\mathcal{V}}\left\langle\tilde{u} \tilde{u}^{*}\right\rangle ; \quad E_{\theta}=\frac{1}{\mathcal{V}}\left\langle\tilde{v} \tilde{v}^{*}\right\rangle ; \quad E_{z}=\frac{1}{\mathcal{V}}\left\langle\tilde{w} \tilde{w}^{*}\right\rangle
$$

with

$$
\langle.\rangle=\int_{R_{1}}^{R_{2}} \int_{0}^{2 \pi} \int_{0}^{\frac{2 \pi}{k}} \operatorname{real}(.) r d r d \theta d z .
$$

The optimal perturbation which is initially in the form of azimuthal streaks transforms progressively through the anti-lift up mechanism (Antkowiak \& Brancher 2007) into azimuthal rolls. This is illustrated by the perturbation equation written for the azimuthal vorticity $\omega_{\theta}^{\prime}=\partial u^{\prime} / \partial z-\partial w^{\prime} / \partial r$. In the inviscid limit, its evolution is governed by

$$
\frac{\partial \omega_{\theta}^{\prime}}{\partial t}=-2 R e_{1} \Omega^{b} \frac{\partial v^{\prime}}{\partial z}
$$

where $\Omega^{b}=V^{b} / r$ is the basic angular velocity. It clearly appears that $\omega_{\theta}^{\prime}$ is driven by the axial gradient of the azimuthal streaks $\frac{\partial v^{\prime}}{\partial z}$. According to Antkowiak \& Brancher (2007), the fluid particles with positive azimuthal streaks undergo an enhanced centrifugal force that will not be balanced by the pressure gradient, and will thus be pushed towards the outer wall. On the contrary, the particles with negative azimuthal streaks will be pushed against the inner wall. This process will initiate the formation of azimuthal rolls.

Outside the Rayleigh line, a stratification of the angular momentum exists and the epicyclic frequency is no longer zero. At initial time, the contours of the kinetic energy associated to the perturbation are oriented against the basic shear, as shown in figure $27(\mathrm{a})$ in plane $(r, z)$ and in figure $27\left(\mathrm{a}^{\prime}\right)$ in plane $(r, \theta)$. These contours will tilt progressively until they almost align with the shear at $t=t^{o p t}$ as shown in figures $27(\mathrm{c})$ and $27\left(c^{\prime}\right)$. 

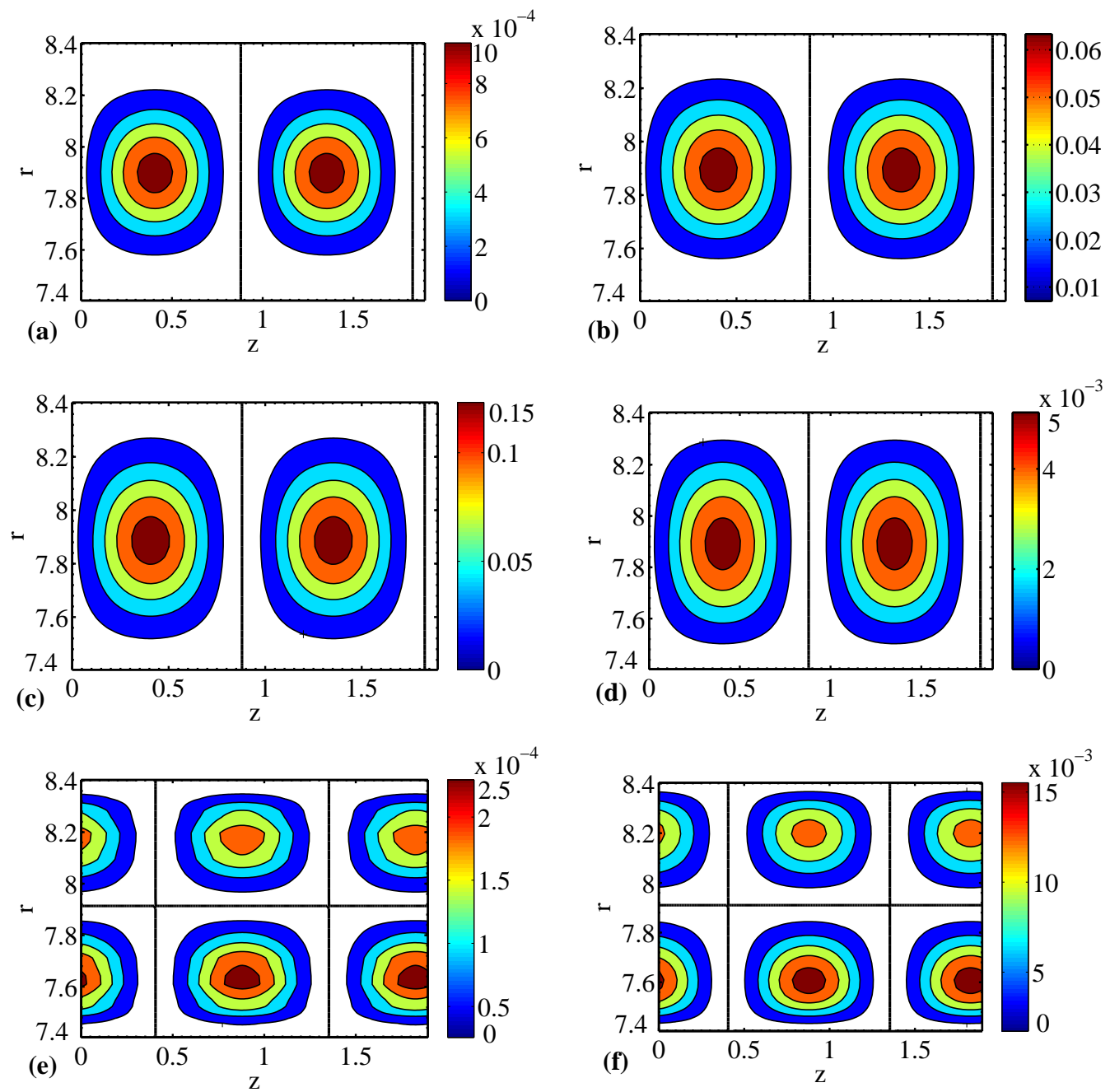

Figure 25. Optimal perturbation represented in plane $(r, z)$ for a Newtonian fluid along the Rayleigh line with $\eta=0.881, R e_{1}=1135, R e_{2}=1000, m^{o p t}=0, k^{o p t}=3.32$ and $G^{o p t}=5.58$. Contours of the radial component of the kinetic energy $1 / 2 u^{\prime 2}(t)$ at $(a)$ initial time $t=0$ and (b) optimal time $t=t^{o p t}$. Contours of the azimuthal component of the kinetic energy $1 / 2 v^{\prime 2}(t)$ at $(c)$ initial time $t=0$ and $(d)$ optimal time $t=t^{o p t}$. Contours of the axial component of the kinetic energy $1 / 2 w^{\prime 2}(t)$ at $(e) t=0$ and $(f)$ optimal time $t=t^{o p t}$.

\subsubsection{Purely viscous shear-thinning fluids}

In the case of purely viscous shear-thinning fluids, the angular momentum is no longer constant along the Rayleigh line. The optimal perturbation in the whole stable domain 


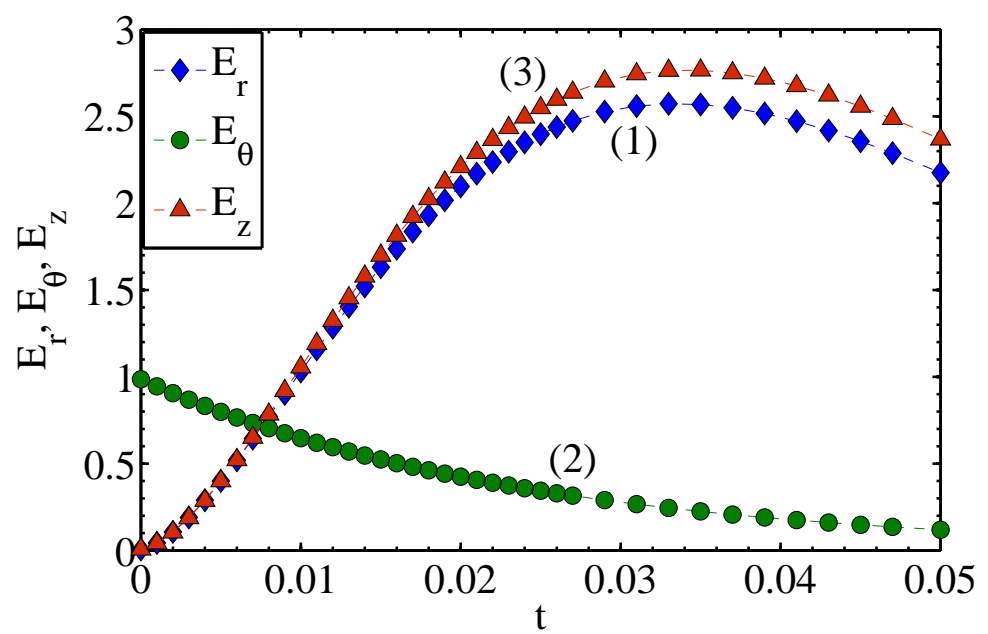

Figure 26. Radial (1), azimuthal (2) and axial (3) kinetic energy components on the Rayleigh line for a Newtonian fluid with $\eta=0.881, m^{o p t}=0, k^{o p t}=3.32, G^{o p t}=5.58, R e_{1}=1135$ and $R e_{2}=1000$

of plane $\left(R e_{1}, R e_{2}\right)$ is qualitatively similar to that obtained for a Newtonian fluid outside the Rayleigh line. Further computations show that the evolution of the optimal perturbation with time for shear-thinning fluids with $\eta=0.881$ and $\eta=0.4$ are similar to the Newtonian one. However, the optimal perturbation is merely concentrated near the inner wall and more pronounced for large gap $\eta=0.4$. Furthermore, the transient growth is reduced with decreasing $\eta$.

\subsubsection{Discussion}

$3 \mathrm{D}$ optimal perturbations are initially oriented in the direction opposite to the base flow and aligned with it at optimal time, e.g. figure $27(c)$ and figure $27\left(c^{\prime}\right)$. The same evolution was observed for other shear flows such as the boundary-layer flow (see Cherubini et al. (2010); Monokrousos et al. (2010) and references therein). This is in contrast with $2 \mathrm{D}$ optimal perturbations which are oriented transversally to the main shear at the optimal time, as shown by Butler \& Farrell (1992) for plane Couette and Poiseuille 

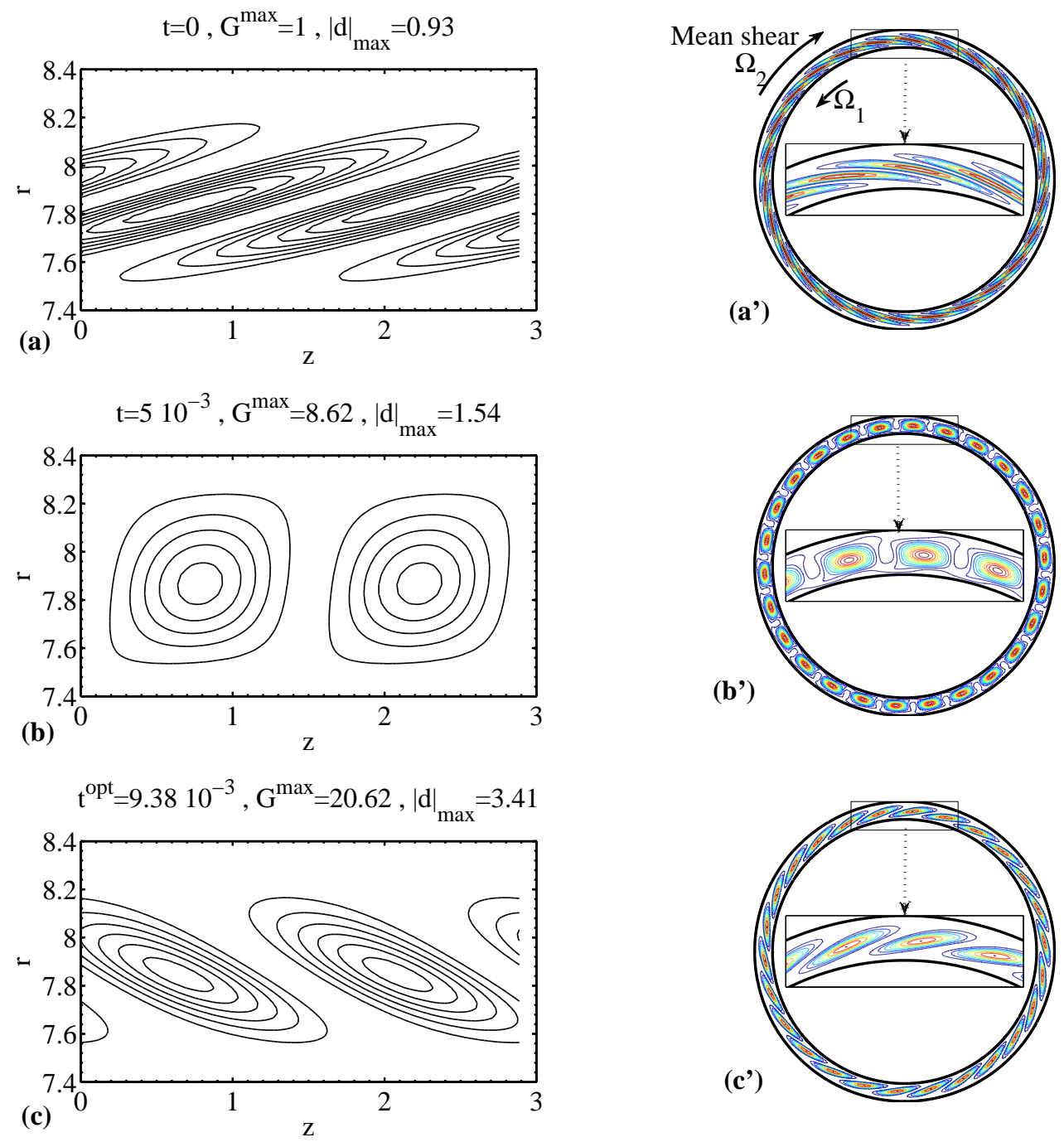

Figure 27. Optimal perturbation for a Newtonian fluid for $\eta=0.881, R e_{1}=101$, $R_{2}=-1061.2$ with $m^{o p t}=13, k^{o p t}=2.176$. Left: contours of the kinetic energy in the plane $(r, z)$ at $(a)$ initial time $t=0,(b) t \approx t^{o p t} / 2$ and $(c)$ optimal time $t=t^{o p t}$. Right: contours of the kinetic energy in the plane $(r, \theta)$ at $\left(a^{\prime}\right)$ initial time $t=0,\left(b^{\prime}\right) t \approx t^{o p t} / 2$ and $\left(c^{\prime}\right)$ optimal time $t=t^{o p t}$. The quantity $d=\partial u^{\prime} / \partial r+u^{\prime} / r+(1 / r) \partial v^{\prime} / \partial \theta$ is the $2 \mathrm{D}$ divergence on the shear plane.

flows. 2D perturbations, i.e., spanwise-independent perturbations, grow only via the Orrmechanism (Orr 1907): a mechanism based on Kelvin's circulation theorem. The initial contour of the vertical structure shortens as it is tilted by the mean shear. As a conse- 

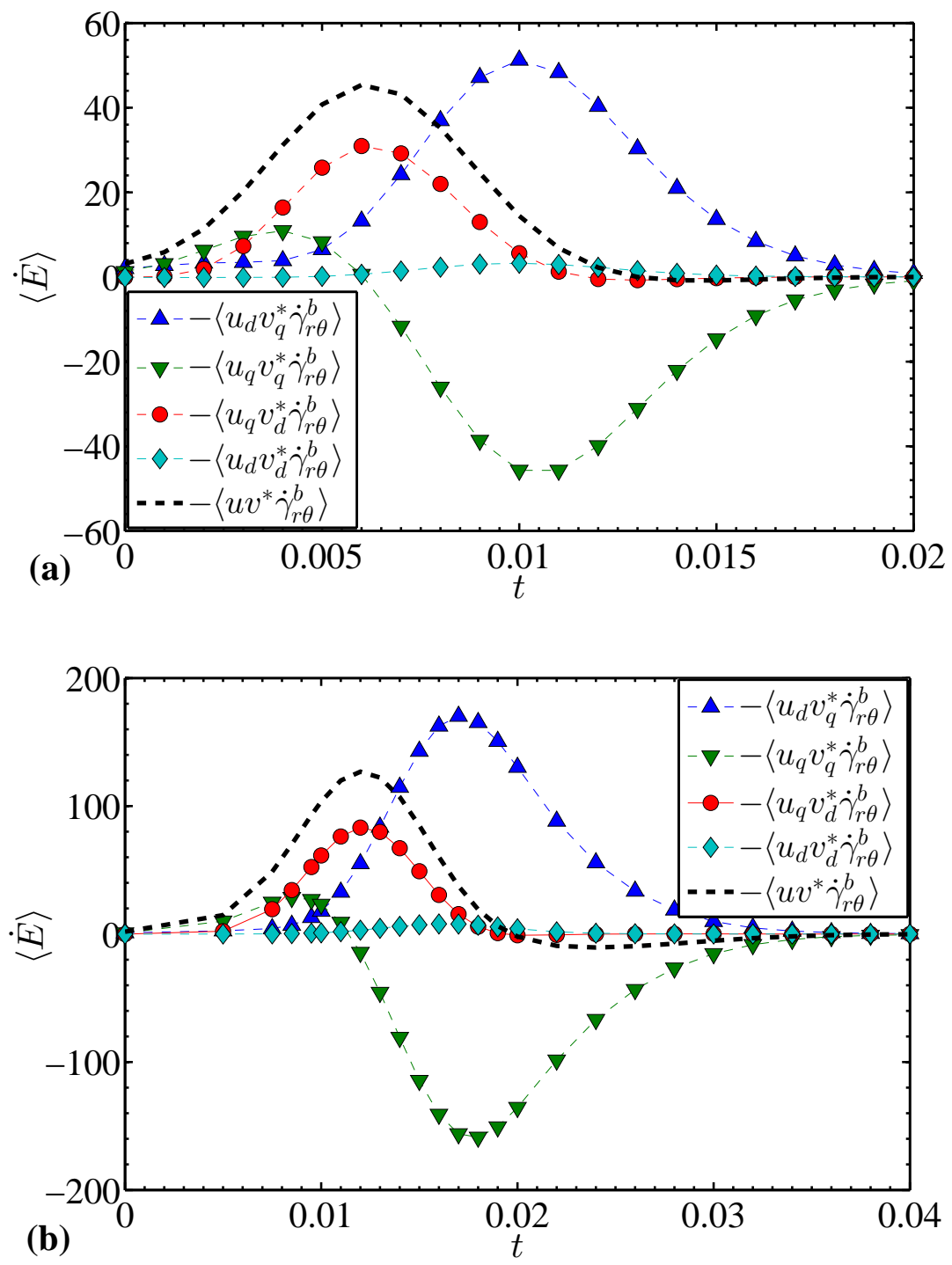

FiguRE 28. Contribution of the different Reynolds stress components to the energy amplification of a 3D perturbation for $\eta=0.881, R e_{1}=101$ and $R e_{2}=-1061.2$. (a) Newtonian fluid, $m^{o p t}=13, k^{o p t}=2.176$ and $G^{o p t}=20.62$. (b) Power-law fluid $n=0.5, m^{o p t}=11, k^{o p t}=1.647$ and $G^{o p t}=88.16$.

quence, the velocity along the contour has to increase to conserve the initial circulation. In $3 \mathrm{D}$ situation, another mechanism, absent in $2 \mathrm{D}$, is active. Indeed, the basic shear vorticity, pointing in the spanwise (here axial) direction, may be stretched due to variations of areas in the shear plane as it is was shown by Vitoshkin et al. (2012) in plane shear 
flows. These authors pointed out that the interplay between the spanwise vorticity $q$ and the planar divergence $d$ fields play a dominant role in energy growth. We extended their analysis to the case of Taylor-Couette flow and found that there is (as in the plane shear flow) a mutual growth of $d$ and $q$ and the maximum is reached at $t=t^{\text {opt }}$, i.e. when the perturbation is tilted with the basic shear. Following Vitoshkin et al. (2012), the role played by the interaction between $d$ and $q$ is highlighted in the analysis of the energy growth via the Reynolds stress term. For this, a 2D Helmholtz decomposition is performed

$$
\tilde{\boldsymbol{u}}=\boldsymbol{u}_{d}+\boldsymbol{u}_{q} \quad \text { with } \quad \boldsymbol{u}_{d}=\boldsymbol{\nabla}(\phi) \quad \text { and } \quad \boldsymbol{u}_{q}=-\boldsymbol{r o t}\left(\psi \boldsymbol{e}_{z}\right)
$$

where $\phi$ and $\psi$ are scalar functions, such that the planar divergence $d=\partial u^{\prime} / \partial r+u^{\prime} / r+$ $(1 / r) \partial v^{\prime} / \partial \theta$ and the axial vorticity $q=(1 / r) \partial\left(r v^{\prime}\right) / \partial r-(1 / r) \partial u^{\prime} / \partial \theta$ satisfy

$$
d=\Delta_{H}(\phi) \text { and } q=\Delta_{H}(\psi)
$$

where $\Delta_{H}=\partial^{2} / \partial r^{2}+(1 / r) \partial / \partial r+\left(1 / r^{2}\right) \partial / \partial \theta^{2}$ is the 2D Laplacian on the shear plane. At the inviscid limit the spatially averaged energy equation is

$$
\begin{aligned}
\frac{\partial\langle E\rangle}{\partial t} & =-R e_{1}\left\langle\dot{\gamma}_{r \theta}^{b} \tilde{u} \tilde{v}^{*}\right\rangle \\
& =-R e_{1}\left\langle\dot{\gamma}_{r \theta}^{b}\left(u_{d}+u_{q}\right)\left(v_{d}^{*}+v_{q}^{*}\right)\right\rangle \\
& =-R e_{1}\left(\left\langle\dot{\gamma}_{r \theta}^{b} u_{d} v_{d}^{*}\right\rangle+\left\langle\dot{\gamma}_{r \theta}^{b} u_{d} v_{q}^{*}\right\rangle+\left\langle\dot{\gamma}_{r \theta}^{b} u_{q} v_{d}^{*}\right\rangle+\left\langle\dot{\gamma}_{r \theta}^{b} u_{q} v_{q}^{*}\right\rangle\right)
\end{aligned}
$$

with $E=\frac{|\tilde{\mathbf{u}}|^{2}}{2}, \boldsymbol{u}_{d}=\left(u_{d}, v_{d}, w_{d}\right)$ and $\boldsymbol{u}_{q}=\left(u_{q}, v_{q}, w_{q}\right)$.

The first term in the R.H.S of (7.15) is the contribution of the divergent field. The second and the third terms, i.e. mixed $q-d$ terms translated the interplay between the planar divergence and the axial vorticity. The fourth term represents the 2D Orr mechanism. The contribution of the different terms as a function of time is shown in figure 28 (a) for a Newtonian fluid and figure 28(b) for a power-law fluid. Qualitatively, evolutions of the different Reynolds stress components are quite similar, however the amplitudes reached 
are higher for power-law fluids. For both fluids, the energy growth is initially dominated by the rotational term $-\left\langle\dot{\gamma}_{r \theta}^{b} u_{q} v_{q}^{*}\right\rangle$, i.e. the $2 \mathrm{D}$ Orr mechanism. From $t \approx t^{o p t} / 2$, the vortices are tilted with the shear, $-\left\langle\dot{\gamma}_{r \theta}^{b} u_{q} v_{q}^{*}\right\rangle$ becomes negative, i.e. Orr mechanism contributes to the decay of the energy. The contribution of the planar divergence term is positive but very small. The contribution of the mixed term $d-q$ is positive and are at the origin of the increase of $E$ when the vortices align with the base shear.

\section{Conclusion}

In this paper, we performed a three-dimensional linear stability analysis of a circular Couette flow of shear-thinning fluids in a thin $(\eta=0.881)$ and a wide $(\eta=0.4)$ gaps between two infinitely coaxial cylinders. Long and short times evolutions of the disturbances is investigated. Two rheological models were adopted, power-law and Carreau models, to describe the shear-thinning behavior of the fluids.

Firstly, we considered the asymptotic evolution at long times. Using a normal-mode approach, an eigenvalue problem was derived and solved with a Chebyshev spectral collocation method. The results show that for a given $R e_{2}$, the shear-thinning character leads to a decrease of the inner critical Reynolds number $R e_{1 c}$ except in the vicinity of $R e_{2}=0$ and for a co-rotating regime with a small gap. This reduction is due to an enhancement of the energy exchange between the perturbation and the base flow, which is probably a consequence of the increase of the shear rate at the inner cylinder wall.

For counter-rotating cylinders, the threshold of the outer Reynolds number, $\left|R e_{2}\right|$ at which the critical modes switches from axisymmetric Taylor vortices to spiral vortices increases significantly with increasing shear-thinning effects. The axial wavelength of these spiral vortices is strongly reduced with increasing shear-thinning effects, because of the reduction of the "effective gap" where the Rayleigh's instability criterion holds. 
Secondly, we studied the transient growth of the perturbation kinetic energy that occurs at short times due to the non-normality of the operators involved in the linearized perturbation equations. The evidence of non-normality was highlighted by plotting $\varepsilon$ pseudospectra. It was shown that the sensitivity of eigenvalues increases with more pronounced shear-thinning character. This effect is confirmed by the strong increase, up to one order of magnitude of the perturbation energy amplification compared to the Newtonian fluid for given $R e_{1}$ and $R e_{2}$.

As far as the structure of the optimal perturbation is concerned, we showed that at large shear Reynolds numbers and for a thin gap, it exhibits a large azimuthal wavenumber $m^{\text {opt }} \approx 12$ and a small axial wavenumber $1 \leqslant k^{\text {opt }} \leqslant 2$. Whereas for a wide gap $(\eta=0.4)$, $m^{\text {opt }}=2$ and $k^{\text {opt }} \propto 1$. It appears that shear-thinning effects have little influence on $k^{\text {opt }}$ and $m^{o p t}$.

- In the particular case of a Newtonian fluid with $\left(R e_{1}, R e_{2}\right)$ on the Rayleigh line, the angular momentum is constant and the optimal perturbation consists of a superposition along the axial direction of high and low azimuthal velocity streaks which evolve towards Taylor vortices via the anti-lift-up mechanism. The optimal energy growth behaves as $G^{o p t} \propto R e^{2}$

- Outside the Rayleigh line, the optimal perturbation, initially oriented against the base shear, tilts until it almost aligns with the shear at $t=t^{\text {opt }}$. The amplification of the $3 \mathrm{D}$ optimal perturbation is analyzed in terms of interplay between the planar divergence and the axial vorticity as suggested by Vitoshkin et al. (2012). The decomposition of the Reynolds stress in terms of the various contributions from the divergent and the rotational fields indicates that the energy growth is dominated by the mixed terms $-\left(u_{d} v_{q}+u_{q} v_{d}\right) \dot{\gamma}_{r \theta}^{b}$ which overwhelms the decaying effect of the Orr-mechanism. The numerical results show that the optimal energy growth behaves as $G_{\text {opt }} \propto R e^{2 / 3}$ in agreement with Maretzke 
et al. (2014).

For shear-thinning fluids; the Rayleigh line should not be distinguished from other situations, the tilting mechanism, described above, applies and the numerical results show that $G^{o p t}=G_{n=1}^{o p t} \times \exp \left(-n^{1 / 3}\right)$. The transient energy growth is enhanced with increasing shear-thinning effects. Thus, shear-thinning promotes transition to turbulence by the linear superposition of eigenmodes. This tendency was observed in a plane Couette flow (Liu \& Liu 2011) and a Hagen-Poiseuille flow (Liu \& Liu 2012) of power-law fluids involving another transient growth mechanism.

\section{Appendix A. Operators involved in the linearized perturbation}

\section{equations}

The initial value problem (5.12) reads

$$
\mathcal{L} \tilde{\boldsymbol{q}}=\mathcal{M} \frac{\partial \tilde{\boldsymbol{q}}}{\partial t}
$$

\section{A.1. $(\tilde{u}, \tilde{v})$ formulation}

In this case, $\tilde{\boldsymbol{q}}=(\tilde{u}, \tilde{v})^{T}$ and the operators $\mathcal{M}$ and $\mathcal{L}$ are expressed below

$$
\begin{aligned}
& \mathcal{M}=\left(\begin{array}{cc}
D D_{*}-k^{2} & \frac{i m}{r} \tilde{D} \\
-\frac{i m}{k^{2} r} D_{*} & 1+\frac{m^{2}}{k^{2} r^{2}}
\end{array}\right) \\
& \mathcal{L}=\mathcal{L}_{I}+\mathcal{L}_{V 1}+\mathcal{L}_{V 2}+\mathcal{L}_{V 3} \\
& \mathcal{L}_{I}=\operatorname{Re}_{1}\left(\begin{array}{cc}
\frac{i m}{r}\left[-\left(V^{b} \widetilde{D}+D V^{b}\right) D_{*}+k^{2} V^{b}\right] & -2 \frac{k^{2} V^{b}}{r}+\frac{m^{2}}{r^{2}}\left(-\frac{2 V^{b}}{r}+D V^{b}+V^{b} D\right) \\
-D_{*} V^{b}-\frac{m^{2}}{k^{2} r^{2}} V^{b} D_{*} & -\frac{i m}{r}\left(1+\frac{m^{2}}{k^{2} r^{2}}\right) V^{b}
\end{array}\right) \\
& \mathcal{L}_{V 1}=\mu^{b}\left(\begin{array}{cc}
\left(D D_{*}-k^{2}\right)^{2} & i m\left[\frac{2 k^{2}}{r^{2}}+D \Delta \frac{1}{r}\left(\Delta-\frac{2 D}{r}+\frac{1}{r^{2}}\right)\right] \\
\frac{i m}{r}\left(-\frac{\Delta D_{*}}{k^{2}}+\frac{2}{r}\right) & D D_{*}-k^{2}+\frac{m^{2}}{k^{2} r^{2}}\left(\Delta-\frac{2 D}{r}+\frac{1}{r^{2}}-k^{2}\right)
\end{array}\right)
\end{aligned}
$$




$$
\begin{array}{r}
Y \text {. Agbessi, B. Alibenyahia, C. Nouar, C. Lemaitre and L. Choplin } \\
\mathcal{L}_{V 2}=D \mu^{b}\left(\begin{array}{cc}
D^{2} D_{*}+\Delta D_{*}-k^{2} D & \frac{i m}{r}\left(\Delta+D^{2}-\frac{4 D}{r}+\frac{3}{r^{2}}\right) \\
-\frac{i m}{k^{2} r} D D_{*} & \left(1+\frac{m^{2}}{k^{2} r^{2}}\right) \widetilde{D}
\end{array}\right) \\
+D^{2} \mu^{b}\left(\begin{array}{cc}
k^{2}+D D_{*} & \frac{i m}{r} \widetilde{D} \\
0 & 0
\end{array}\right) \\
\mathcal{L}_{V 3}=\left(\mu_{t}-\mu^{b}\right)\left(\begin{array}{cc}
\frac{k^{2} m^{2}}{r^{2}} & -\frac{i k^{2} m \widetilde{D}}{r} \\
\frac{i m D_{*}}{r} & \left(D+\frac{2}{r}\right) \\
D
\end{array}\right)+D\left(\mu_{t}-\mu^{b}\right)\left(\begin{array}{cc}
0 & 0 \\
\frac{i m}{r} & \widetilde{D}
\end{array}\right)
\end{array}
$$

\section{A.2. $(\tilde{u}, \tilde{w})$ formulation}

In this case, $\tilde{\boldsymbol{q}}=(\tilde{u}, \tilde{w})^{T}$ and the operators $\mathcal{M}$ and $\mathcal{L}$ are expressed below

$$
\begin{aligned}
& \mathcal{M}=\left(\begin{array}{cc}
D_{*}^{2}+\frac{1}{r} D_{*}-\frac{m^{2}}{r^{2}} & i k D+\frac{2 i k}{r} \\
k D_{*} & i k^{2}+\frac{i m^{2}}{r^{2}}
\end{array}\right) \\
& \mathcal{L}=\mathcal{L}_{I}+\mathcal{L}_{V 1}+\mathcal{L}_{V 2}+\mathcal{L}_{V 3} \\
& \mathcal{L}_{I}=\operatorname{Re}_{1}\left(\begin{array}{cc}
\frac{i m}{r^{2}}\left(2 D V^{b}+V^{b} D-3 V^{b} D_{*}+\frac{m^{2} V^{b}}{r}\right) & \frac{3 k m V^{b}}{r^{2}} \\
\frac{i k m}{r}\left(D V^{b}-V^{b} D\right) & \frac{m}{r}\left(\frac{m^{2} V^{b}}{r^{2}}+k^{2} V^{b}\right)
\end{array}\right) \\
& +\operatorname{Re}\left(\begin{array}{cc}
\frac{i m}{r}\left[-\frac{D V^{b}}{r}+D^{2} V^{b}-V^{b} D D_{*}\right] & \frac{k m}{r}\left(V^{b} D+D V^{b}\right) \\
0 & 0
\end{array}\right) \\
& \mathcal{L}_{V 1}=\mu^{b}\left(\begin{array}{cc}
-\frac{2 m^{2}}{r^{4}} & i k\left[\left(\frac{2}{r}+D\right)\left(D_{*} D-k^{2}\right)+\frac{2 D_{*} D}{r}-\left(\frac{4}{r}+D\right) \frac{m^{2}}{r^{2}}\right] \\
k\left(\Delta+\frac{2 D}{r}\right) D_{*} & i k^{2}\left(\Delta+\frac{2 D}{r}\right)
\end{array}\right) \\
& +\mu^{b}\left(\begin{array}{cc}
\left(\frac{2}{r}+D\right) \Delta D_{*}+\frac{2}{r}\left(D^{2}+\frac{D}{r}\right) D_{*}-\frac{m^{2}}{r^{2}} \Delta-\frac{m^{2}}{r^{4}} & 0 \\
\frac{2 k m^{2}}{r^{3}} & 0
\end{array}\right)
\end{aligned}
$$




$$
\begin{aligned}
& \mathcal{L}_{V 2}=D \mu^{b}\left(\begin{array}{cc}
\frac{2}{r} D D_{*}-\frac{m^{2}}{r^{2}}\left(D-\frac{2}{r}\right) & i k\left[\Delta+\left(\frac{4}{r}+D\right) D\right] \\
k D D_{*} & i\left(k^{2}+\frac{m^{2}}{r^{2}}\right) D
\end{array}\right) \\
& +D \mu^{b}\left(\begin{array}{cc}
{\left[\left(D+\frac{2}{r}\right) D+\Delta\right] D_{*}} & 0 \\
0 & 0
\end{array}\right) \\
& +D^{2} \mu^{b}\left(\begin{array}{cc}
D D_{*}+\frac{m^{2}}{r^{2}} & i k D \\
0 & 0
\end{array}\right) \\
& \mathcal{L}_{V 3}=\left(\mu_{t}-\mu^{b}\right)\left(\begin{array}{cc}
{\left[\left(\frac{5}{r}+D\right) D+\frac{3}{r^{2}}+\frac{2 m^{2}}{r^{2}}\right] D D_{*}+\frac{m^{4}}{r^{4}}} & i k\left[\frac{m^{2}}{r^{2}}+\frac{3}{r^{2}}+\left(\frac{5}{r}+D\right) D\right] D \\
k\left[\left(\frac{3}{r}+D\right) D+\frac{m^{2}}{r^{2}}\right] D_{*} & i k^{2}\left(D+\frac{3}{r}\right) D
\end{array}\right) \\
& +D\left(\mu_{t}-\mu^{b}\right)\left(\begin{array}{cc}
\left(\frac{5}{r}+2 D\right) D D_{*}+\frac{m^{2}}{r^{2}}\left(D+D_{*}\right) & i k\left(\frac{5}{r}+2 D\right) D \\
\frac{k m^{2}}{r^{2}}+k D D_{*} & i k^{2} D
\end{array}\right) \\
& +D^{2}\left(\mu_{t}-\mu^{b}\right)\left(\begin{array}{cc}
D D_{*}+\frac{m^{2}}{r^{2}} & i k D \\
0 & 0
\end{array}\right)
\end{aligned}
$$

\section{Appendix B. Demonstration that the eigenvalues for a Newtonian}

\section{fluid on the Rayleigh line are real}

The eigenvalue problem reads

$$
\mathcal{L} \boldsymbol{q}=\sigma \mathcal{M} \boldsymbol{q}
$$

where $\boldsymbol{q}=(u, v)^{T}$ or $(u, w)^{T}$ depending on the formulation adopted.

For a Newtonian fluid, on the Rayleigh line, the angular momentum of the base flow is constant $\frac{d}{d r}\left(r V^{b}\right)^{2}=0$, thus

$$
V^{b}=\frac{a}{r},
$$

where $a$ is a constant scalar and

$$
D_{*} V^{b}=D V^{b}+\frac{V^{b}}{r}=0 .
$$


As stressed by Gebhardt \& Grossmann (1993), two types of modes exist. The first type corresponds to $v \neq 0$ and the second to $v=0$.

- If $v \neq 0$ then the $v$ equation of the $(u, v)$ formulation is considered (second line of system (B 1)),

$$
\sigma v=\mu^{b}\left(D D_{*} v-k^{2} v\right)
$$

This equation is multiplied by the complex conjugate of $v, v^{*}$ and integrated over volume $\mathcal{V}=2 \pi^{2}\left(R_{2}^{2}-R_{1}^{2}\right) / k$

$$
\sigma \int_{R_{1}}^{R_{2}} \int_{0}^{2 \pi} \int_{0}^{\frac{2 \pi}{k}} v v^{*} r d r=\mu^{b} \int_{R_{1}}^{R_{2}} \int_{0}^{2 \pi} \int_{0}^{\frac{2 \pi}{k}}\left(D D_{*} v-k^{2} v\right) v^{*} r d r
$$

After integrations by parts, this equation becomes

$$
\sigma \int_{R_{1}}^{R_{2}} v v^{*} r d r=-\mu^{b} \int_{R_{1}}^{R_{2}}\left[\left(D_{*} v\right)\left(D_{*} v^{*}\right)+k^{2} v v^{*}\right] r d r .
$$

- If $v=0$ then the $u$ equation of the $(u, v)$ formulation is considered (first line of system (B 1)),

$$
\sigma\left(D D_{*}-k^{2}\right) u=\mu^{b}\left(D D_{*}-k^{2}\right)^{2} v
$$

This equation is multiplied by the complex conjugate of $u, u^{*}$ and integrated over a volume $\mathcal{V}$ and after integrations by parts, we obtain

$-\sigma \int_{R_{1}}^{R_{2}}\left[\left(D_{*} u\right)\left(D_{*} u^{*}\right)+k^{2} u u^{*}\right] r d r=\mu^{b} \int_{R_{1}}^{R_{2}}\left(D D_{*} u-k^{2} u\right)\left(D D_{*} u^{*}-k^{2} u^{*}\right) r d r$.

For both equations (B 8) and (B 6), the coefficient of the eigenvalue $\sigma$ and the right hand side are real and do not depend on $R e_{1}$. Furthermore, the left hand side coefficient is negative and the right hand side is positive. The eigenvalue is thus real and negative and is independent of $R e_{1}$. 

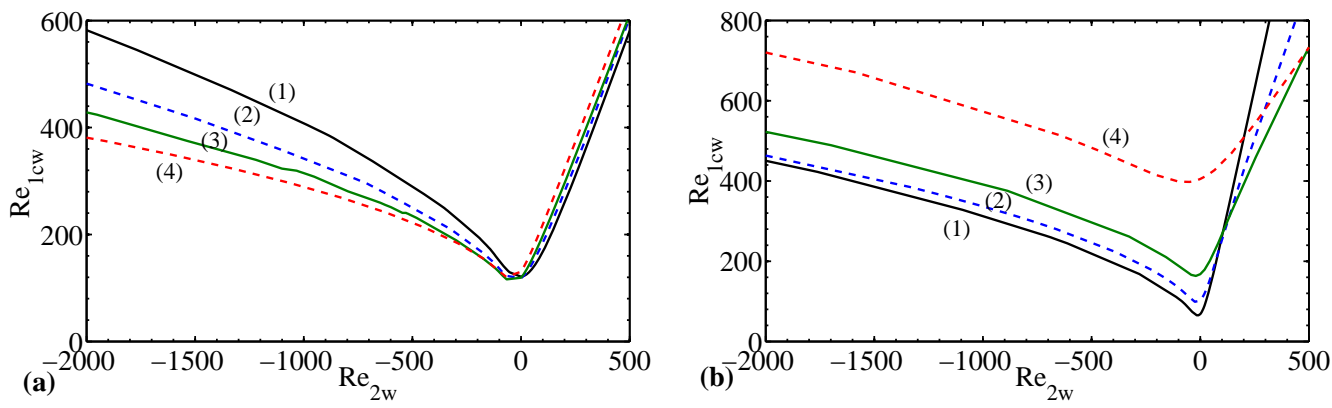

FIgURE 29. Marginal stability curves in the plane $\left(R e_{1 w}, R e_{2 w}\right)$ for a power-law fluid: (1)

$n=1$, Newtonian fluid, (2) $n=0.7$, (3) $n=0.5$, and (4) $n=0.3$. (a) $\eta=0.881$; (b) $\eta=0.4$.

\section{Appendix C. Effect of the viscosity scale on the}

\section{stabilization/destabilization of shear-thinning}

We adopt here a definition of the Reynolds numbers based on the viscosity evaluated at the inner wall, $\hat{\mu}^{b}\left(\hat{R}_{1}\right)$

$$
\operatorname{Re}_{(1,2) w}=\frac{\hat{\rho} \hat{R}_{1} \hat{\Omega}_{(1,2)} \hat{d}}{\hat{\mu}^{b}\left(\hat{R}_{1}\right)} .
$$

Figure 29 shows marginal stability curves in terms of $R e_{1 w}$ and $R e_{2 w}$,

$$
R e_{1 w}=\frac{\hat{\rho} \hat{R}_{1} \hat{\Omega}_{1} \hat{d}}{\hat{\mu}^{b}\left(\hat{R}_{1}\right)}, \quad R e_{2 w}=\frac{\hat{\rho} \hat{R}_{2} \hat{\Omega}_{2} \hat{d}}{\hat{\mu}^{b}\left(\hat{R}_{1}\right)} .
$$

It appears that the shear-thinning behavior has a destabilizing effect for a thin gap $\eta=0.881$ when both cylinders are counter-rotating. However, for a large gap $\eta=0.4$, stabilization is observed. The opposite effect is found by using as scale the viscosity defined by (2.9). The selection of the viscosity scale may be considered to be simply a matter of choice, however the conclusions that one reaches by comparing different shearthinning fluids among themselves and against Newtonian fluids can be radically different from one choice to the other. 


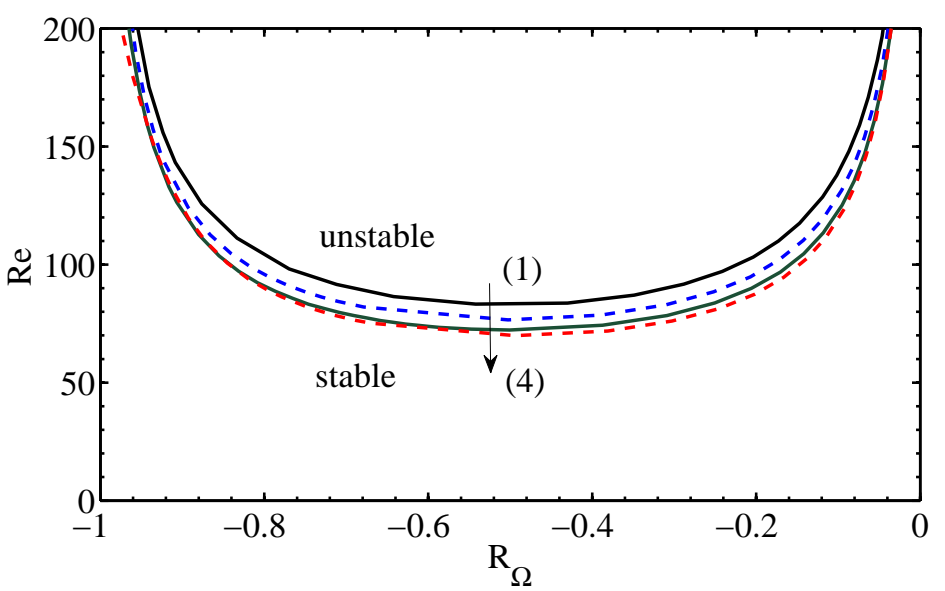

FIGURE 30. Marginal stability curves in the plane $\left(R e, R_{\Omega}\right)$ for a power-law fluid with $\eta=0.881$. (1) Newtonian fluid, (2) $n=0.7$, (3) $n=0.5$, and (4) $n=0.3$.
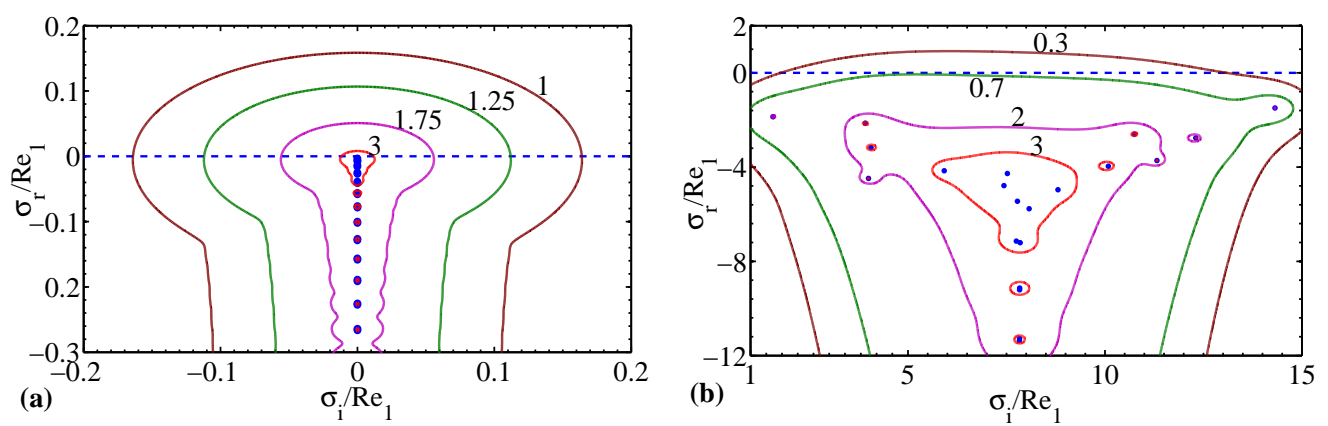

Figure 31. Pseudospectrum for a Newtonian fluid with $\eta=0.881$. (a) Rayleigh line with $R e_{1}=6.30 \times 10^{3}, R e_{2}=5.55 \times 10^{3}, k=3.32, m=0$; (b) counter-rotating cylinders with $R e_{1}=101, R e_{2}=-1061.2, k=2.176, m=13$.

\section{Appendix D. Marginal stability for a power-law fluid in the plane}

$$
\left(R e, R_{\Omega}\right)
$$

Figure 30 shows marginal stability curves for a power-law fluid in the plane $\left(R e, R_{\Omega}\right)$. For a Newtonian fluid, the marginal stability curve is delimited by the lines $R_{\Omega}=-1$ and $R_{\Omega}=(1-\eta) / \eta$. 

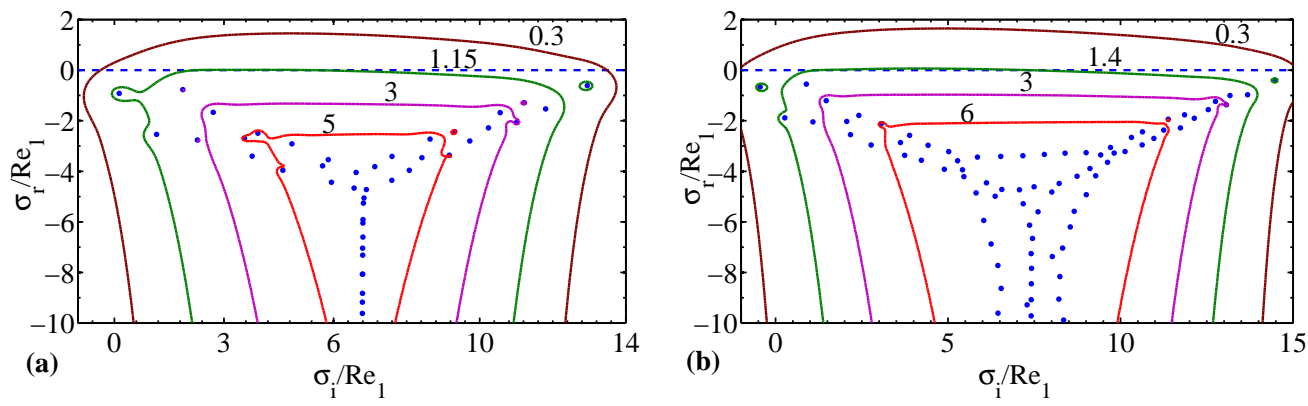

FiguRE 32. Pseudospectra for shear-thinning fluids with $R e_{1}=101, R e_{2}=-1061.2$ and $\eta=0.881$. (a) Power-law fluid with $n=0.5, k=1.647, m=11$; (b) Carreau fluid with $n=0.5$, $\lambda=10, k=1.842, m=12$.

\section{Appendix E. Non-normality and pseudospectra}

Let $\mathscr{C}$ be the discrete representation of $\mathscr{L}$. The $\varepsilon$-pseudospectrum of $\mathscr{C}$ is defined as the set of complex numbers $z$ for which $\left\|(z I-\mathscr{C})^{-1}\right\|_{E}^{2}>\varepsilon^{-1}$, or equivalently, the set of complex numbers $z$ which are eigenvalues of $\mathscr{C}+\Delta \mathscr{C}$ for some perturbation matrix $\Delta \mathscr{C}$ with $\|\Delta \mathscr{C}\|_{E}^{2}<\varepsilon$, where $\|(.)\|_{E}^{2}$ is the energy norm defined by equation (7.1). It is usually displayed graphically with contours of the norm of the resolvent $(z I-\mathscr{L})$ for various values of $\varepsilon$. The more non-normal the linear operator $\mathscr{L}$ is, the greater is the potential of a disturbance operator $\Delta \mathscr{C}$ to affect the eigenvalues.

Figure 31 (a) displays the spectral portrait of operator $\mathscr{L}$ for a Newtonian fluid, on the Rayleigh line with $k=3.32, m=0, R e_{1}=6.30 \times 10^{3}, R e_{2}=5.55 \times 10^{3}$. The iso-contours labeled $p$ delineate the boundaries in the complex plane of the $\varepsilon$-pseudospectrum with $\varepsilon=10^{-p}$. Non-normality effects are more pronounced for eigenvalues near the origin. For the given values of $R e_{1}$ and $R e_{2}$, a perturbation of $\mathrm{O}\left(10^{-3}\right)$ allows the pseudospectrum to protrude into the unstable region. Figure 31(b) shows the spectral portrait of the Taylor-Couette flow outside the Rayleigh line. Non-normal effects are more pronounced at the intersection of the three branches of the spectrum. A perturbation of $\mathrm{O}\left(10^{-0.7}\right)$ is required to extend the pseudospectrum into the unstable half plane. For axisymmetric 
perturbations, non-normality phenomena are strongly reduced. For the same $R e_{1}$ and $R e_{2}$ non-normality effects are more important for shear-thinning fluids, figure 32. For instance, for a Carreau fluid with $n=0.5$ and $\lambda=10$, a perturbation of $\mathrm{O}\left(10^{-1.4}\right)$ is necessary to extend the pseudospectrum into the unstable region.

\section{REFERENCES}

Alibenyahia, B., Lemaitre, C., Nouar, C. \& Ait-Messaoudene, N. 2012 Revisiting the stability of circular Couette flow of shear-thinning fluids. J. Non - Newtonian. Fluid Mech. 183, 37-51.

Altmeyer, S., Hoffmann, Ch. \& Lucke, M. 2011 Islands of instability for growth of spiral vortices in the Taylor-Couette system with and without axial through flow. Phys. Rev. E. 84, 046308, 1-12.

Andereck, C.D., Liu, S.S. \& Swinney, H.L. 1986 Flow regimes in a circular Couette system with independently rotating cylinders. J. Fluid Mech. 164, 155-183.

Antkowiak, A. \& Brancher, P. 2007 On vortex rings around vortices: an optimal mechanism. J. Fluid Mech. 578, 295-304.

Ashrafi, N. \& Khayat, R. E. 2000 Shear-induced chaos in Taylor-vortex flow. Phys. Rev. E. 61, 1455-1467.

AttA, C. VAN 1966 Exploratory measurements in spiral turbulence. J. Fluid Mech. 25, 495-512.

Billant, P. \& Gallaire, F. 2005 Generalized Rayleigh criterion for non-axisymmetric centrifugal instabilities. J. Fluid Mech. 542, 365-379.

Bird, R., Amstrong, R. \& Hassager, O. 1987 Dynamics of polymeric liquids. Wiley - Interscience, New York.

Butler, K. M. \& Farrell, B. M. 1992 Three - dimensional optimal perturbations in viscous shear flow. Phys. Fluids A 4, 1637-1650.

Carreau, J. P. 1972 Rheological equations from molecular network theories. J. Rheol. 16, $99-127$. 
CAton, F. 2006 Linear stability analysis of circular Couette flow of inelastic viscoplastic fluids. J. Non-Newtonian Fluid Mech. 134, 148-154.

Cherubini, S., Robinet, J.-C., Bottaro, A. \& De Palma, P. 2010 Optimal wave packets in a boundary layer and initial phases of a turbulent spot. J. Fluid Mech. 656, 231-259.

Coles, D. 1965 Transition in circular Couette flow. J. Fluid Mech. 21, 385-425.

Coronado-Malutti, O., Mendes, P. R. Souza \& Carvallo, M. S. 1986 Instability of inelastic shear-thinning liquids in a Couette flow between concentric cylinders. Chem. Eng. Sci. 41, 2915-2923.

DiPrima, R. C. \& Hall, P. 1984 Complex eigenvalues for the stability of couette flow. Proc. Roy. Soc. of Lond.. A. Mathematical and Physical Sciences 396 (1810), 75-94.

Drazin, P.G. \& ReID, W.H. 1981 Hydrodynamic stability. In Cambridge Mathematical Library. Cambridge University Press.

Dubrulle, B., Dauchot, O., Daviaud, F., Longaretti, P.Y., Richard, D. \& Zhan, J.P. 2005 Stability and turbulent transport in Taylor-Couette flow from analysis of experimental data. Phys. Fluids. 17, 095103, 1-19.

Escudier, M.P., Gouldson, I.W. \& Jones, D.M. 1995 Taylor vortices in Newtonian and shear-thinning liquids. Proc. Roy. Soc. A 449, 155-176.

Gebhardt, Thomas \& Grossmann, Siegfried 1993 The Taylor - Couette eigenvalue problem with independently rotating cylinders. Physik B Condensed Matter 90 (4), 475-490.

Gebhart, T. \& Grossmann, S. 1993 The Taylor-Couette eigenvalue problem with independently rotating cylinders. Condensed Matter. 90, 475-490.

GiesEKUs, H. 1966 Zur Stabilität von Strömungen viskoelastischer Flüssigkeiten. 1.Ebene und kreisförmige Couette-Strömung. Rheol. Acta 5, 239-252.

Groisman, A. \& Steinberg, V. 1998 Mechanism of elastic instability in Couette flow of polymer solutions: Experiments. Phys. Fluids. 10, 2451-2463.

Hristova, H., Roch, S., Schmid, P. \& Tuckerman, S.L. 2002 Transient growth in TaylorCouette flow. Phys. Fluids. 14, 3475-3484.

Jastrzebski, M., Zaidani, H.A. \& Wronski, S. 1992 Stability of Couette flow of liquids with power-law viscosity. Rheol. Acta 31, 264-273. 
Koschmieder, E.L. 1993 Bénard Cells and Taylor Vortices. . In Cambridge Monographs on Mechanics and Applied Mathematics. Cambridge University Press, New York.

Krueger, E.R., Gross, A. \& Diprima, R.C. 1966 On the relative importance of Taylorvortex and non-axisymmetric modes in flow between rotating cylinders. J. Fluid Mech. 24, $521-538$.

Langford, F., Tagg, R., Kostelich, E., Swinney, H.L. \& Glubitsky, M. 1988 Primary instabilities and bicriticality in flow between counter rotating cylinders. Phys. Fluids. 31, $776-785$.

Larson, R. G., Shaqfeh, E.S.G. \& Muller, S.J. 1990 A purely elastic instability in TaylorCouette flow. J. Fluid Mech. 210, 573-600.

LiU, R. \& LiU, Q. S. 2011 Non-modal instability in plane Couette flow of a power law fluid. J. Fluid Mech. 676, 145-171.

LiU, R. \& LiU, Q. S. 2012 Non-modal stability in Hagen-Poiseuille flow of a shear-thinning fluid. Phys. Rev. E 85, 066318.

Lockett, T.J., Richardson, S.M. \& Worraker, W.J. 2004 The stability of inelastic nonNewtonian fluids in Couette flow between concentric cylinders. J. Fluid. Eng. 126, 385-390.

Maretzke, S., Hof, B. \& Avila, M. 2014 Transient growth in linearly stable Taylor-Couette flows. J. Fluid Mech. 742, 254-290.

Meseguer, A. 2002 Energy transient growth in the Taylor-Couette problem. Phys. Fluids. 14, 1655-1660.

Monokrousos, Antonios, Akervik, Espen, Brandt, Luca \& Henningson, Dan S. 2010 Global three-dimensional optimal disturbances in the blasius boundary - layer flow using time-steppers. J. Fluid Mech. 650, 181-214.

Muller, S.J., Larson, R.G. \& Shaqfeh, E.S.G. 1989 A purely elastic transition in TaylorCouette flow. Rheol. Acta 24, 499-503.

OrR, W. M'F 1907 The stability or instability of the steady motions of a perfect liquid and of a viscous liquid. Proc. R. Irish Acad. 27, 9-138.

Pascal, J. P. \& Rasmussen, H. 1995 Stability of power law fluid flow between rotating cylinders. Dynam. Stabil. Systm. 10, 65-93. 
Ranganathan, B. T. \& Govindarajan, R. 2001 Stabilization and destabilization of channel flow by location of viscosity - stratified fluid layer. Phy. Fluids 13, 1-3.

Reddy, Satish C., Schmid, Peter J. \& Henningson, Dan S. 1993 Pseudospectra of the Orr - Sommerfeld Operator. SIAM J. Appl. Math 53, 15-47.

Schmid, P. J. \& Henningson, D. S. 2001 Stability and transition in shear flows, Applied Mathematical Sciences, vol. 142. Springer.

Shaqfeh, E.S.G., Muller, S.J. \& Larson, R.G. 1992 The effect of gap width and dilutesolution properties on the viscoelastic Taylor-Couette instability. J. Fluid Mech. 235, 285317.

Shu, F. H. 1982 The Physical Universe: An Introduction to Astronomy. University Science Books.

Sinevic, V., Kuboi, R. \& Nienow, A.W. 1986 Power numbers, Taylor numbers and Taylor vortices in viscous Newtonian and non-Newtonian fluids. Chem. Eng. Sci. 41, 2915-2923.

SNyder, H.A. 1968 Stability of a rotating Couette flow. i Asymmetric waveform. Phys. Fluids. 11, $728-734$.

TAGG, R. 1994 The Couette-Taylor problem. Nonlinear Science Today 4, 1-25.

TAnneR, R. 2000 Engineering rheology. Oxford University Press, New York.

TAYLOR, G. I. 1923 Stability of a viscous liquid contained between two rotating cylinders. Phil. Trans. R. Soc. London. A 223, 289-343.

Trefethen, L. N., Chapman, S. J. R., Henningson, D. S., Meseguer, A., Mullin, T. \& Nieuwstadt, F. T. M. 2000 Threshold amplitudes for transition to turbulence in a pipe. Numerical Analysis Report 00/17.

Trefethen, Lloyd N., Trefethen, Anne E., Reddy, Satish C. \& Driscoll, Tobin A. 1993 Hydrodynamic stability without eigenvalues. Science 261 (5121), 578-584.

Vitoshikin, H., Heifetz, E., Gelfgat, A. Yu. \& Harnik, N. 2012 On the role of vortex stretching in energy optimal growth of three - dimensional perturbations on plane parallel shear flows. J. Fluid Mech. 707, 369-380. 